\title{
ON THE REAL SPECTRUM OF A RING AND ITS APPLICATION TO SEMIALGEBRAIC GEOMETRY
}

\author{
BY EBERHARD BECKER
}

Introduction. This paper is meant as an introduction and a guide to some recent developments in real algebraic geometry - more precisely, in semialgebraic geometry. In real algebraic geometry one is concerned with the set of real points $V(\mathbf{R})$ of a variety $V$ defined over $\mathbf{R}$. More generally, one may replace the field of real numbers $\mathbf{R}$ by any real closed field. Real algebraic geometry is clearly a part of general algebraic geometry and therefore there seems to be no need for special considerations, i.e. special notions, tools, etc. However, in dealing with the set of real points $V(\mathbf{R})$ one encounters new phenomena which are not, or at least not easily, treatable by the general methods of algebraic geometry. To give examples, let $V$ be an affine variety over $\mathbf{R}$. Then $V(\mathbf{R})$ can be regarded as an algebraic subset of some suitable $\mathbf{R}^{N}$, i.e., a subset defined by a finite set of polynomial equations $F_{1}=0, \ldots, F_{r}=0$ where $F_{i} \in$ $\mathbf{R}\left[X_{1}, \ldots, X_{N}\right], i=1, \ldots, r$. Consequently, $V(\mathbf{R})$ carries the subspace topology inherited from $\mathbf{R}^{N}$. Even if $V$ is irreducible it may happen that $V(\mathbf{R})$ is not a connected topological space. Note that the corresponding set of complex points $V(\mathbf{C})$ is always connected if $V$ is irreducible. A typical example is provided by the elliptic curve $E$ (Figure 1).

In this example, $E(\mathbf{R})$ has two components $C_{1}, C_{2}$, namely

$$
\begin{aligned}
& C_{1}=\left\{(x, y) \in \mathbf{R}^{2} \mid y^{2}=x\left(x^{2}-1\right), x \leqslant 0\right\}, \\
& C_{2}=\left\{(x, y) \in \mathbf{R}^{2} \mid y^{2}=x\left(x^{2}-1\right), x \geqslant 1\right\},
\end{aligned}
$$

We notice that the components are described by equalities and inequalities. This is quite generally true: $V(\mathbf{R})$ always has a finite number of components each of which can be described by a finite number of polynomial equalities and inequalities, cf. [Lo, Wh].

Thus, one is naturally led to consider subsets of $V(\mathbf{R})$ which can be described by finitely many polynomial equalities and inequalities: these are the so-called semialgebraic subsets of $V(\mathbf{R})$.

Semialgebraic subsets of $V(\mathbf{R})$ or $\mathbf{R}^{N}$ arise in the above-mentioned study of components. However, they are to be considered as the natural objects of study in real algebraic geometry not only because of this occurrence. Their definition takes account of the entire structure of the real numbers as an ordered field.

Received by the editors August 29, 1985.

1980 Mathematics Subject Classification (1985 Revision). Primary 14G30, 12D15.

(C)1986 American Mathematical Society $0273-0979 / 86 \$ 1.00+\$ .25$ per page 


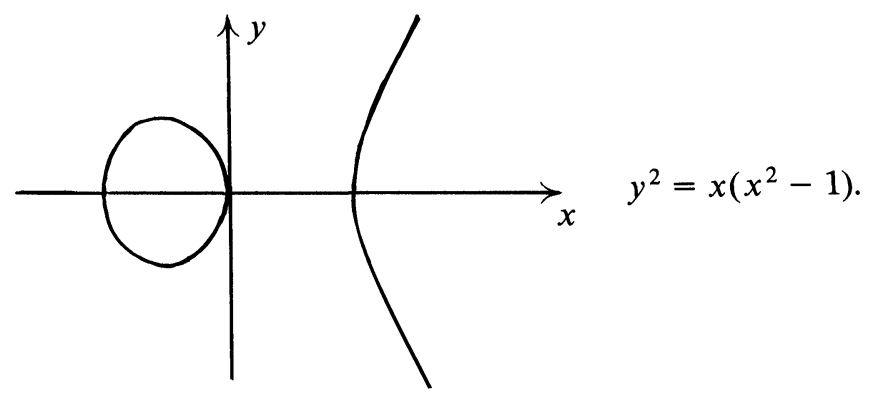

FIGURE 1

Since an algebraically closed field cannot be ordered there is no similar notion over the complex field. It is the order of $\mathbf{R}$ which enables one to define interior and exterior of figures, e.g. the interior of circles, polygons, etc.

So one may say that semialgebraic sets are in some respects the genuine objects of real algebraic geometry. Consequently, real algebraic geometry should be extended to include the investigation of semialgebraic sets, i.e. it should be extended to semialgebraic geometry. A good account of currently studied problems in semialgebraic geometry can be found in the proceedings [CT-C-M-R, DR], in Brumfiel's book [Bru1], in volume 14, number 4 of the Rocky Mountain Journal (1984), and in the book of Delfs and Knebusch [DK3].

As explained, semialgebraic geometry deals with problems which do not completely fit in classical algebraic geometry. This raises the question: Are there suitable notions and tools to treat these problems properly? It is the conviction of several mathematicians including the author, that the appropriate notion has been found. It is the new notion of the real spectrum of a ring which was introduced by M. Coste and M. F. Coste-Roy about 1979 [CC, CC , $_{1}$, CR]. In this concept ideas from the Zariski-spectrum of a ring are cleverly combined with concepts from the theory of formally real fields. That this latter theory naturally plays a role in real algebraic geometry was already obvious in Artin's solution of Hilbert's 17th problem. Today it seems that the notion of the real spectrum of a ring may serve as a building block for a general semialgebraic geometry in the same way that the Zariski spectrum of a ring did for Grothendieck's formulation of algebraic geometry. In particular, it will certainly stimulate the further development of real commutative algebra.

Let $A$ denote any commutative ring with unit. Given a prime ideal $\mathfrak{y}$ its residue field quot $(A / \mathfrak{y})$ is denoted by $k(\mathfrak{y})$. As a set, the real spectrum of $A$, denoted by $\mathscr{R}$-Spec $A$, consists of all pairs $(\mathfrak{y}, \bar{P})$ where $\mathfrak{y} \in \operatorname{Spec} A$ and $\bar{P}$ is an order in $k(\mathfrak{y})$. The topology is defined as follows: Given $a \in A$, set $D(a)=\{(\mathfrak{y}, \bar{P}) \mid a \notin \mathfrak{y}, a+\mathfrak{y} \in \bar{P}\}$. Now by definition, these sets $D(a)$, $a \in A$, constitute a subbasis for the topology. In this topology, $\mathscr{R}$-Spec $A$ turns 
out to be a quasicompact space. Moreover, the assignment $A \mapsto \mathscr{R}$-Spec $A$ is, in a natural way, a contravariant functor from the category of commutative rings with unit into the category of topological spaces.

As mentioned above, the notion of the real spectrum was introduced by Coste and Coste-Roy. They were led to it by topos-theoretic considerations. In this present paper, we will follow a different approach. We start off with Artin's solution of the 17 th problem. Today, the result is usually derived from the Artin-Lang homomorphism theorem. In the first section the notion of the real spectrum is developed from an analysis of this theorem. One may even say that this approach provides a clearer understanding of the Artin-Lang theorem.

The second section is devoted to a general study of the real spectrum as a topological space and as a functor. In the third section we shall deal with certain applications to semialgebraic geometry. Here, we are concerned with an affine variety $M$ defined over a real closed field $R$ and semialgebraic subsets of $M(R)$, the set of real points of $M$. The semialgebraic subsets of $M(R)$ are in one-to-one correspondence with the constructible subsets of $\mathscr{R}$-Spec $R[M]$, where $R[M]$ denotes the coordinate ring of $M$. A constructible set in $\mathscr{R}$-Spec $A$ is any subset which can be obtained from the $D(a)$ 's, $a \in A$, as above, by taking a finite number of intersections, unions and complements. That this correspondence is a bijection is essentially equivalent to the Artin-Lang theorem, and it is used in particular, following v. d. Dries, to prove the so-called finiteness theorem of semialgebraic geometry. This theorem states that any open semialgebraic set can be defined by a finite number of strict inequalities $f_{1}>0, \ldots, f_{s}>0$ where $f_{i} \in R[M]$. Bröcker's recent results on the number of the inequalities needed are explained.

In the last section, we shall discuss the use of the real spectrum of a ring for a possible foundation of an "abstract" semialgebraic geometry. The main part of this section is concerned with the construction of a certain sheaf on any constructible subset $X$ in the real spectrum of a ring. This sheaf is called the sheaf of abstract semialgebraic functions on $X$ and was independently introduced by G. Brumfiel and N. Schwartz. $X$ together with this sheaf (or possibly another, cf. $[\mathbf{R}]$ ) may serve as the building block of an abstract semialgebraic geometry.

There are other introductions to the theory of the real spectrum, cf. [CC1, L2, Kn]. They are all highly recommended. The reader will notice that different points of view are taken in these papers, and different notations are used. The notation $\mathscr{R}-\operatorname{Spec} A$, used in this paper, is chosen to reflect the fact that one may understand the theory of the real spectrum as a theory of ring homomorphisms into real closed fields. In a corresponding manner the Zariski-spectrum $\operatorname{Spec} A$ is related to homomorphisms into algebraically closed fields. Generalizing these two examples, one may specify a class $\mathscr{K}$ of fields and consider only homomorphisms from a ring $A$ into fields belonging to $\mathscr{K}$. This would then lead to a $\mathscr{K}$-spectrum of $A$, naturally denoted by $\mathscr{K}$-Spec $A$. In the case of the real spectrum, $\mathscr{R}$ means the class of real closed fields. A notation like $\mathscr{K}$-Spec $A$ seems flexible enough to cover further examples. At present, it is 
already clear that one has to study the formally $p$-adic spectrum of a ring in order to globalize the results of [PR], cf. [BS].

The forthcoming book of Bochnak, Coste and Roy will present semialgebraic geometry in great detail. The author hopes that this present paper will stimulate interest in semialgebraic geometry and that the reader will pass on to this comprehensive book.

1. From the solution of Hilbert's 17th problem to the real spectrum. In this section we first recall the solution of Hilbert's 17th problem which is based on the Artin-Lang homomorphism theorem. This latter theorem provides, in fact, more than the solution of this famous problem (which is, of course, already important enough). Indeed, it leads directly to defining the real spectrum, $\mathscr{R}$-Spec $A$, of a ring $A$. Using this notion, a better understanding of the Artin-Lang homomorphism theorem can be obtained.

We will not be concerned with the 17th problem in its full generality, nor in its original form; see the paper of McKenna [Mc] for a comprehensive discussion. We fix a real closed field $R$ and we will deal with the following version:

Let the polynomial $f \in R\left[X_{1}, \ldots, X_{n}\right]$ be positive on $R^{n}$, i.e. $f(x) \geqslant 0$ for every $x \in R^{n}$. Is $f$ then a sum of squares of rational functions in $R\left(X_{1}, \ldots, X_{n}\right)$ ?

As is well known, E. Artin answered this question in the affirmative [A] (in fact he dealt with a slightly different situation). He first studied sums of squares in arbitrary fields $K$. Set $\sum K^{2}=\left\{\sum_{1}^{r} x_{i}^{2} \mid r \in \mathbf{N}, x_{1}, \ldots, x_{r} \in K\right\}$. If char $K=2$ then $\sum K^{2}=K^{2}$; if char $K \neq 2$ but $-1 \in \sum K^{2}$ then $\sum K^{2}=K$ because of the identity $a=((a+1) / 2)^{2}+(-1)((a-1) / 2)^{2}$. Hence, the fields with the property $-1 \notin \sum K^{2}$ are left. These fields are called formally real and for those fields Artin proved [A] the

(1.1) Proposition. $\sum K^{2}=\bigcap P$ where $P$ ranges over all orders of $K$.

Recall that an order $P$ of $K$ is any subset of $K$ satisfying $P+P \subset P$, $P P \subset P, P \cup-P=K, P \cap-P=\{0\}$, in other words, the orders are just the cones of positivity of the total order relations on $K$.

The proof of (1.2) is easy and can be found, e.g., in [W, P, L1].

In the next step of his proof Artin had to show that a polynomial $f$, positive on $R^{n}$, lies in every order $P$ of the rational function field $R\left(X_{1}, \ldots, X_{n}\right) ;(1.1)$ would then give the desired conclusion $f \in \sum R\left(X_{1}, \ldots, X_{n}\right)^{2}$. Today, this is often derived from the so-called Artin-Lang homomorphism theorem [La, p. 279 , Theorem 5], for affine $R$-algebras which are, by definition, nothing but the finitely generated commutative $R$-algebras. We will proceed in this manner.

(1.2) ThEOREM. Let $A$ be an affine R-algebra which is a domain and denote its quotient field by $L$. If $L$ is formally real (or, equivalently, if the unique order of $R$ can be extended to $L$ ) then there is an R-algebra homomorphism $\varphi: A \rightarrow R$.

In order to apply this result we start with the function field $L_{0}=$ $R\left(X_{1}, \ldots, X_{n}\right)$ and $f \in A_{0}=R\left[X_{1}, \ldots, X_{n}\right]$ which is assumed not to be a sum of squares in $L_{0}$. By (1.1), we find an order $P$ with $f \notin P$, hence $-f \in P$. Now 
consider the function field $L=L_{0}(\sqrt{-f})$ which is the quotient field of $A=$ $A_{0}[1 / f, \sqrt{-f}]$. It is also formally real since the assumption $-1 \in \sum L^{2}$, say $-1=\sum\left(a_{i}+b_{i} \sqrt{-f}\right)^{2}, a_{i}, b_{i} \in L_{0}$, would imply $\left(\sum b_{i}^{2}\right) f=1+\sum a_{i}^{2}$ and finally $f \in \sum L_{0}^{2} \subset P$. By (1.1) we therefore get a homomorphism $\varphi: A \rightarrow R$. We have $\varphi(f) \neq 0$ since $f$ is a unit of $A$. We then obtain $\varphi(-f)=\varphi(\sqrt{-f})^{2}>0$, hence $\varphi(f)=f\left(\varphi\left(X_{1}\right), \ldots, \varphi\left(X_{n}\right)\right)<0$, which means that $f$ is strictly negative at the point $\left(\varphi\left(X_{1}\right), \ldots, \varphi\left(X_{n}\right)\right) \in R^{n}$. Thus we have solved, following E. Artin, the 17 th problem of Hilbert.

It was the application of (1.2) to the larger function field $L$ and the algebra $A$ instead of $R\left(X_{1}, \ldots, X_{n}\right)$ and $R\left[X_{1}, \ldots, X_{n}\right]$ that brought the contradiction. This flexibility with respect to the variation of the "parameters" $L$ and $A$ allows a version of the Artin-Lang homomorphism theorem, which immediately points to a description of orderings by ultrafilters. To this end, we introduce a few algebraic-geometric notions: Let $A$ be an affine $R$-algebra, for simplicity assume $A=R\left[X_{1}, \ldots, X_{n}\right] / \mathfrak{a}$. We attach to $A$ the real affine algebraic set $M(R)=\left\{x \in R^{n} \mid F(x)=0\right.$ for all $\left.F \in \mathfrak{a}\right\}$. The algebra $A$ has a natural representation as a ring of $R$-valued functions on $M(R)$ : if $f=F+$ a, $F \in R\left[X_{1}, \ldots, X_{n}\right]$ and $x \in M(R)$, then $f(x):=F(x)$. We have the natural map

$$
\left\{\begin{array}{l}
M(R) \rightarrow \operatorname{Spec} A, \\
x \mapsto \mathfrak{m}_{x}=\{f \in A \mid f(x)=0\} .
\end{array}\right.
$$

It is easily verified that this map is injective, and the image is just the set of maximal ideals with $R$ as their residue field. Consequently, $M(R)$ is referred to as the set of real points of the affine scheme $M=\operatorname{Spec} A$.

A point $\mathfrak{y} \in \operatorname{Spec} A$, i.e. a prime ideal, is called regular if the local ring $A_{\mathfrak{y}}$ is a regular local ring [K]. Accordingly $x \in M(R)$ is called regular if its local ring $\mathcal{O}_{x}:=A_{\mathfrak{m}_{x}}$ is regular. The existence of regular points is crucial, as we shall see. We set $M(R)_{\text {reg }}:=\{x \in M(R) \mid x$ regular $\}$.

EXAMPLES. (i) $A=\mathbf{R}[X, Y] /\left(X^{2}+Y^{2}+1\right), M(\mathbf{R})=\varnothing$.

(ii) $A=\mathbf{R}[X, Y] /\left(X^{2}+Y^{2}\right), M(\mathbf{R})=\{(0,0)\}, M(\mathbf{R})_{\mathrm{reg}}=\varnothing$.

(iii) $A=\mathbf{R}[X, Y] /\left(Y^{2}-X^{3}\right), M(\mathbf{R})_{\mathrm{reg}}=M(\mathbf{R}) \backslash\{(0,0)\}, M(\mathbf{R})$ :

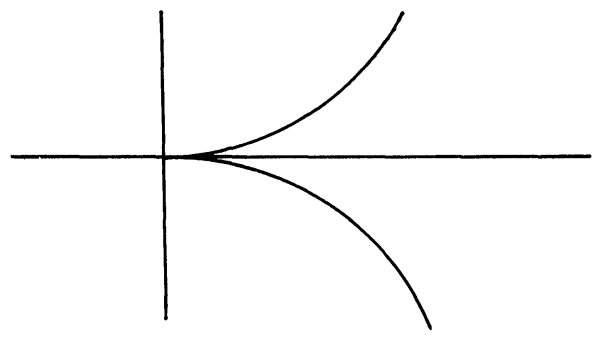

The following variant of the Artin-Lang homomorphism theorem was proved in $[B,(1.3)]$. The statement seems much stronger than that of (1.2). But in fact, it is a consequence of (1.2) and, in turn, implies the previous formulation (1.2). To see this take $n=1, f_{1}=1$. In comparing the formulation of the next theorem with that of loc. cit. (1.3) one should note that $M(R) \rightarrow \operatorname{Hom}_{R}(A, R)$, 
$x \mapsto\{f \mapsto f(x)\}$ is a bijection, where $\operatorname{Hom}_{R}(A, R)$ is the set of all unitary $R$-algebra homomorphisms from $A$ to $R$.

(1.3) THEOREM. Let $L$ be the formally real quotient field of the affine $R$-algebra $A$ and let $f_{1}, \ldots, f_{n} \in A \backslash\{0\}$ be given. Then the following statements are equivalent:

(i) There exists a regular real point $x \in M(R)$ with $f_{1}(x)>0, \ldots, f_{n}(x)>0$.

(ii) There is an order $P$ of $L$ with $f_{1}, \ldots, f_{n} \in P$.

The equivalence of the two statements (i) and (ii) indicates a close link between the two notions of positivity for a function $f \in A$, namely being abstractly positive, i.e. lying in some order, and being positive as a function at some point. It is this connection we wish to pursue. For this purpose, we need further notions. The real closed field $R$ is clearly a topological field. We therefore have a topology on $R^{n}$ and consequently the subspace topology on $M(R)$. This topology is referred to as the strong topology. A neighborhood basis for a point $x \in M(R)$ is given by the balls $B(x, \varepsilon) \cap M(R), \varepsilon \in R$, $\varepsilon>0$, where $y \in B(x, \varepsilon)$ iff $d(x, y)^{2}=\sum_{1}^{n}\left|x_{i}-y_{i}\right|^{2}<\varepsilon^{2}$.

We further have the Zariski topology on $\operatorname{Spec} A$ [Bo2]. A basis for this topology is given by the sets $U(f)=\{\mathfrak{y} \in \operatorname{Spec} A \mid f \notin \mathfrak{y}\}$; the closed sets are given by $V(\mathfrak{a})=\{\mathfrak{y} \in \operatorname{Spec} A \mid \mathfrak{a} \subset \mathfrak{y}\}$, $\mathfrak{a}$ an ideal of $A$. Via the embedding $M(R) \hookrightarrow \operatorname{Spec} A$ we get the subspace Zariski topology on $M(R)$ with a basis given by $\{x \in M(R) \mid f(x) \neq 0\}, f \in A$. This second topology is of course coarser than the strong topology on $M(R)$. In general it is strictly coarser, as can be seen from the example $A=R[X], M(R)=R$, where the nonempty Zariski-open sets are just the complements of the finite sets.

The real spectrum $\mathscr{R}-\operatorname{Spec} A$ will remedy this defect. We will have mappings

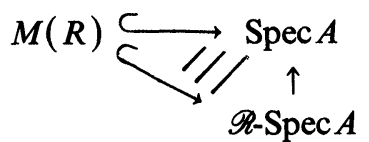

such that the subspace topology on $M(R)$ inherited from $\mathscr{R}-\operatorname{Spec} A$ is just the strong topology on $M(R)$.

For the moment, however, we are dealing with the Zariski topology on $\operatorname{Spec} A$ and the natural embedding $M(R) \hookrightarrow \operatorname{Spec} A$. We will need to describe the closure of a set $S \subset M(R)$ in $\operatorname{Spec} A$.

Given $\mathfrak{y} \in \operatorname{Spec} A$ we denote by $k(\mathfrak{y})$ the residue field on $\mathfrak{y}$, i.e. the quotient field of $A / \mathfrak{y}$. We have $k(\mathfrak{y})=A_{\mathfrak{y}} / \mathfrak{y} A_{\mathfrak{y}}$. Now let $S \subset M(R)$ be given, its closure in the Zariski topology of $\operatorname{Spec} A$ is denoted by $\bar{S}^{z}$. It is obtained as follows: if $S=\varnothing$ then $\bar{S}^{z}=\varnothing$, if $S \neq \varnothing$ then $I(S):=\bigcap_{x \in S} \mathfrak{m}_{x}$, is a radical ideal in the noetherian ring $A$, hence $I(S)=\mathfrak{y}_{1} \cap \cdots \cap \mathfrak{y}_{r}$ where the $\mathfrak{y}_{i}$ are all the minimal prime ideals of $I(S)$. Note that this intersection is irredundant.

(1.4) Proposition. $\bar{S}^{z}=V\left(\mathfrak{y}_{1}\right) \cup \cdots \cup V\left(\mathfrak{y}_{r}\right)$. Moreover, $k\left(\mathfrak{y}_{i}\right)$ is formally real for $i=1, \ldots, r$.

PROof. By the general theory of $\operatorname{Spec} A$ we have $\bar{S}^{z}=V(I(S))=\bigcup_{1}^{r} V\left(\mathfrak{y}_{i}\right)$. To show that all $k\left(\mathfrak{y}_{i}\right)$ are formally real we have to prove: if $\Sigma_{1}^{s} f_{j}^{2} \in \mathfrak{y}_{i}$ then $f_{1}, \ldots, f_{s} \in \mathfrak{y}_{i}$. For simplicity, set $i=1$. Pick $g \in\left(\mathfrak{y}_{2} \cap \cdots \mathfrak{y}_{r}\right) \backslash \mathfrak{y}_{1} ;$ then 
$\sum_{1}^{s}\left(g f_{j}\right)^{2} \in \mathfrak{y}_{1} \cap \cdots \cap \mathfrak{y}_{r}=I(S)$. This means $\sum\left(g f_{j}\right)(x)^{2}=0$ for all $x \in S$, hence $g f_{j} \in I(S)$ for all $j$ 's. Since $g \notin \mathfrak{y}_{1}$ we get $f_{j} \in \mathfrak{y}_{1}$ for $j=1, \ldots, s$.

$S$ is called Zariski-dense if $\bar{S}^{z}=\operatorname{Spec} A$. In case $\operatorname{Nil}(A)=0$ we have

(1.5) $S$ is Zariski-dense if and only if: $f=0$ on $S$ implies $f=0$.

The basic result in this regard is the following one.

(1.6) Proposition. Assume that $A$ is an integral domain. Then every subset $S \subset M(R)$ which is open in the strong topology and contains a regular point is Zariski-dense.

This was first proved by Dubois and Efroymson [DE, p. 134, Theorem 4.9]. The proof as given in [B, p. 8, (1.5)] only uses the Artin-Lang homomorphism theorem and is valid over an arbitrary real closed field.

Now we are almost prepared to state and prove the first part of the ultrafilter theorem. We only need the notion of a semialgebraic set in $M(R)$. Given arbitrary finitely many elements $f, g_{1}, \ldots, g_{r} \in A$ we set

$$
\begin{aligned}
\left\{f=0, g_{1}>0, \ldots, g_{r}>0\right\} & \\
: & =\left\{x \in M(R) \mid f(x)=0, g_{i}(x)>0, i=1, \ldots, r\right\} .
\end{aligned}
$$

Then, by definition, a semialgebraic set is a finite union of sets of the type $\left\{f=0, g_{1}>0, \ldots, g_{r}>0\right\}$. Intuitively, a set is semialgebraic (for short: s.a.) if it can be described by a finite number of equalities and inequalities. One might wonder why only one equation occurs in $\left\{f=0, g_{1}>0, \ldots, g_{r}>0\right\}$. The reason is that the statement $f_{1}=0, \ldots, f_{s}=0$ is equivalent to $\Sigma_{1}^{s} f_{i}^{2}=0$. Note that there is no such way to combine several inequalities. Examples for this are easily provided in the case of $A=R[M]$. The semialgebraic subsets of $M(R)$ form a lattice $\gamma(M(R))$ closed even with respect to taking the complement. It is the lattice, with complement, which is generated by the sets $\{f>0\}, f \in A$. As in any lattice there are filters in $\gamma(M(R))$, and the maximal filters are called ultrafilters. Here, slightly generalizing the definition in [Bi, p. 25], a filter in a lattice $\mathscr{L}$ is any dual ideal $\mathscr{J} \neq \mathscr{L}$.

We now can state the ultrafilter theorem for orders of a function field. It is due to Brumfiel [Bru1, p. $232 \mathrm{ff}$.]. Let $L$ be a function field over $R$, which is the quotient field of an affine $R$-algebra $A$. Given an order $P$ of $L$ we assign to $P$ the filter $\mathscr{F}(P)$ in $\gamma(M(R))$ which is generated (as a filter) by the semialgebraic subsets $\left\{f_{1}>0, \ldots, f_{r}>0\right\}$ of $M(R)$, where $r \in \mathbf{N}, f_{i} \in A$, $f_{i} \in P \backslash\{0\}$ for $i=1, \ldots, r$. Conversely, given any filter $F$ in $\gamma(M(R))$ set

$$
\mathscr{P}(F)=\left\{f \in L \mid f g^{2} \in A \text { and }\left\{f g^{2} \geqslant 0\right\} \in F \text { for some } g \in A \backslash\{0\}\right\} .
$$

(1.7) Ultrafilter TheORem For Orders. The mapping $P \mapsto \mathscr{F}(P)$ is a bijection, with inverse $F \rightarrow \mathscr{P}(F)$, between the set of orders of $L$ and the set of ultrafilters in $\gamma(M(R))$ which contain only Zariski-dense semialgebraic subsets of $M(R)$.

Proof. Let the order $\boldsymbol{P}$ be given. The sets $\left\{f_{1}>0, \ldots, f_{r}>0\right\}$ are clearly open in the strong topology. Hence, by (1.3) and (1.6) $\mathscr{F}(P)$ contains only Zariski-dense sets. In order to show that $\mathscr{F}(P)$ is an ultrafilter we have to 
prove that any semialgebraic subset $T$ of $M(R)$ satisfies $T \in \mathscr{F}(P)$ or $M(R) \backslash$ $T=: T^{c} \in \mathscr{F}(P)$. Suppose $T^{c} \notin \mathscr{F}(P)$. We want to show $T \in \mathscr{F}(P)$. To this end we may assume that $T$ is of the form $T=\left\{f=0, g_{1}>0, \ldots, g_{r}>0\right\}$. If $f \neq 0$ we would get $f^{2} \in A \cap P,\left\{f^{2}>0\right\} \subset T^{c}$, thus $T^{c} \in \mathscr{F}(P)$, which is a contradiction. Therefore $f=0$, and from $T^{c} \notin \mathscr{F}(P)$ we derive $g_{1}, \ldots, g_{r} \in P$ yielding $T \in \mathscr{F}(P)$.

Conversely let $F$ be an ultrafilter of Zariski-dense semialgebraic subsets of $M(R)$. If $f_{1}, f_{2} \in P:=\mathscr{P}(F)$, and with $g_{i} \in A \backslash\{0\}, f_{i} g_{i}^{2} \in A,\left\{f_{i} g_{i}^{2} \geqslant 0\right\} \in$ $\mathscr{F}$, then

$$
\left\{f_{i} g_{i}^{2} \geqslant 0\right\} \subset\left\{f_{i}\left(g_{1} g_{2}\right)^{2} \geqslant 0\right\}
$$

and

$$
\left\{f_{1} g_{1}^{2} \geqslant 0\right\} \cap\left\{f_{2} g_{2}^{2} \geqslant 0\right\} \subset\left\{f\left(g_{1} g_{2}\right)^{2} \geqslant 0\right\},
$$

where $f$ denotes $f_{1}+f_{2}$ or $f_{1} f_{2}$. This shows $\left\{f\left(g_{1} g_{2}\right)^{2} \geqslant 0\right\} \in \mathscr{F}$ and $P+P$ $\subset P, \quad P P \subset P$. If $f \in P \cap-P$ then $\left\{f g^{2} \geqslant 0\right\} \in F,\left\{-f h^{2} \geqslant 0\right\} \in F$, so $\left\{f(g h)^{2}=0\right\} \in F$. By assumption, $F$ contains only Zariski-dense subsets, which implies, in view of (1.5), that $f(g h)^{2}=0$ and $f=0$. To show $P \cup-P$ $=L$ take $f \in L, f \neq 0$ and choose $g \in A \backslash\{0\}$ with $f g^{2} \in A$. Because of $M(R)=\left\{f g^{2}>0\right\} \dot{\cup}\left\{f g^{2}=0\right\} \dot{\cup}\left\{-f g^{2}>0\right\} \in F$ we see that at least one of these three semialgebraic subsets must lie in $F$. By the above argument, $\left\{f g^{2}=0\right\} \in F$ is impossible, from which we get $f \in P \cup-P$.

It remains to show that the mappings $P \mapsto \mathscr{F}(P), F \mapsto \mathscr{P}(F)$ are inverse to each other. If $P$ is given then, for any $f \in A \cap P$, we have $\{f \geqslant 0\} \in \mathscr{F}(P)$. Thus $A \cap P \subset \mathscr{P}(\mathscr{F}(P))$, showing $P=\mathscr{P}(\mathscr{F}(P))$. If $F$ and $f \in \mathscr{P}(F) \cap A$ are given then, as above, $\left\{f g^{2} \geqslant 0\right\} \in F$ implies $f=0$ or $\left\{f g^{2}>0\right\} \in F$. As $\left\{f g^{2}>0\right\} \subset\{f>0\}$, we get $\{f>0\} \in F$. Hence $\mathscr{F}(\mathscr{P}(F)) \subset F$, yielding $\mathscr{F}(\mathscr{P}(F))=F$.

The ultrafilters $\mathscr{F}(P)$ consist of subsets of the topological space $M(R)$ with the strong topology. It is rather natural to look at limit points of those filters. These filters $\mathscr{F}(P)$ are not filters with respect to the power set of $M(R)$, they are, following [Bo1], a filter basis or prefilters. Nevertheless, we have the notion of a limit point in the following sense: given any filter basis $F$ in a topological space $X$ we call $x \in X$ a limit point of $F$ if $x \in \cap_{S \in F} \bar{S}$. In our situation we first see that a filter $\mathscr{F}(P)$ has at most one limit point. Assume on the contrary that $x, y \in R^{n}$ are distinct limit points and (distance) $)^{2}=$ $d(x, y)^{2}=\sum_{1}^{n}\left(x_{i}-y_{i}\right)^{2}=\varepsilon$. Setting $f(z)=\frac{1}{2} \varepsilon-d(z, x)^{2}$ for $z \in M(R)$, we have constructed $f \in A$ with $f(x)>0>f(y)$. If $f \in P$ then $\{f>0\} \in \mathscr{F}(P)$ and, using the assumption, $f(y) \geqslant 0$. If $f \notin P$ one will equally derive a contradiction, this time $f(x) \leqslant 0$.

It may happen that $\mathscr{F}(P)$ has no limit point on $M(R)$ at all. In case $\lim \mathscr{F}(P)=x \in M(R)$ exists we call $x$ the center of $P: x=c(P)$.

To describe the center points we will make use of certain valuation rings. Because of later applications we will introduce them in a more general way than really needed here. Assume $L$ is any ordered field and $B$ is a local subring of $L$ whose maximal ideal $\mathfrak{m}$ is convex in $B$ with respect to the order 
of $L$. By definition this means that $0<a<b, a \in B, b \in \mathfrak{m}$ implies $a \in \mathfrak{m}$. Now let $\hat{B}$ be the convex closure of $B$ in $L$, i.e.

$$
\hat{B}=\{a \in L|| a \mid \leqslant \alpha \text { for some } \alpha \in B\} .
$$

We further set

$$
\hat{\mathrm{m}}=\{a \in L|| a \mid<1 /(1+\alpha) \text { for all } \alpha \in B, \alpha \geqslant 0\} .
$$

In order to formulate the next result we follow the convention that, given two local rings $B_{1}, B_{2}$ in a field, $B_{1}$ is said to be dominated by $B_{2}$ (or: $B_{2}$ dominates $B_{1}$ ) if $B_{1} \subset B_{2}$ and $\mathfrak{m}_{1}=B_{1} \cap \mathfrak{m}_{2}$ where $\mathfrak{m}_{i}$ is the maximal ideal of $B_{i}$. In this case we write $B_{1} \prec B_{2}$.

(1.8) Proposition. Assume the above hypothesis, then

(i) $\hat{B}$ is a valuation ring with maximal ideal $\hat{\mathrm{m}}$,

(ii) $B$ is dominated by $\hat{B}$,

(iii) the residue field $\hat{B} / \hat{\mathrm{m}}$ is archimedean over $B / \mathfrak{m}$ under the induced order which is defined as follows: $\varepsilon+\hat{\mathrm{m}}>0$ iff $\varepsilon>0$.

Proof. That $\hat{B}$ is a ring is easily seen. Let $a \notin \hat{B}$. Then $|a|>\alpha$ for all $\alpha \in B, \alpha \geqslant 0$. Note that $1+\alpha$ is a unit of $B$ if $\alpha \in B, \alpha \geqslant 0$ since $0<1 \leqslant$ $1+\alpha$ and $m$ is convex. Hence, we get $|a|>1+\alpha$ and $\left|a^{-1}\right|<1 /(1+\alpha)$, in particular $a^{-1} \in \hat{B}$. Thus $\hat{B}$ is a valuation ring, the maximal ideal $\hat{\mathrm{m}}$ of which is contained in the set $\{a \in L|| a \mid<1 /(1+\alpha)$ for all $\alpha \in B, \alpha \geqslant 0\}$, as just proved. Conversely, if $|a|<1 /(1+\alpha)$ for all $\alpha \in B, \alpha \geqslant 0$, then $\left|a^{-1}\right|>1+\alpha$ for all $\alpha \in B, \alpha \geqslant 0$, which shows $a^{-1} \notin \hat{B}$, hence $a \in \hat{\mathrm{m}}$. From the convexity of $\mathfrak{m}$ and the description of $\hat{m}$ we get that $\hat{B}$ dominates $B$. The set $\{\varepsilon+\hat{\mathfrak{m}} \mid \varepsilon \in \hat{B}, \varepsilon \geqslant 0\}=: \bar{P}$ is closed under addition and multiplication and we have $\bar{P} \cup-\bar{P}=\hat{B} / \hat{\mathrm{m}}$. If $-1 \in \bar{P}$ then $1+\varepsilon \in \hat{\mathrm{m}}$ for some $\varepsilon \in \hat{B}, \varepsilon \geqslant 0$. But $1+\varepsilon$ is a unit, as seen above, which is a contradiction. Thus $\bar{P}$ is an order of $\hat{B} / \hat{\mathfrak{m}}$. From the definition of $\hat{B}$ one gets the remaining statement in (iii).

Before applying the result (1.8) we would like to point out that it constitutes the essential part of Brumfiel's place extension theorem [Bru1, p. 152, (7.7.4)].

We now return to the description of the center points. We take as $L$ our function field over the real closed field $R$ together with the given order $P$ and as $B$ the subfield $R$. In this case $\hat{R}$ is usually denoted by $A(P, R)$ and $(\hat{0})$ by $I(P, R)$, see [L1, PC].

(1.9) Proposition. Let an order $P$ of $L$ and $x \in M(R)$ be given. Then the following statements are equivalent:

(i) $x$ is the center of $P$.

(ii) $\mathcal{O}_{x}$ is dominated by $A(P, R)$.

Proof. (i) $\Rightarrow$ (ii) Consider $f \in A$, assume $f(x)>0$. If $f \notin P$ then $\{-f>0\}$ $\in \mathscr{F}(P), x \in \overline{\{f<0\}}$, hence $f(x) \leqslant 0$. We thus have that $f(x)>0$ implies $f \in P$. Now let $f$ be any element of $A$. Set $\varepsilon=1+|f(x)|$; then $(\varepsilon \pm f)(x)>0$, consequently $\varepsilon \pm f \in P, f \in A(P, R)$, and $A \subset A(P, R)$. If $f(x)=0$ we get $(\eta \pm f)(x)>0, \eta \pm f \in P$ for all $\eta \in R, \eta>0$, hence $\mathfrak{m}_{x} \subset I(P, R)$ and $\mathcal{O}_{x} \prec A(P, R)$.

(ii) $\Rightarrow$ (i) The distance function $D: M(R) \rightarrow R, y \mapsto d(x, y)^{2}$, lies in $\mathfrak{m}_{x}$, hence $\varepsilon \pm D \in P$ for all $\varepsilon>0$. This means that the open ball $B(x, \sqrt{\varepsilon})$ in 
$M(R)$ lies in $\mathscr{F}(P)=F$, therefore $B(x, \sqrt{\varepsilon}) \cap S \neq \varnothing$ for $S \in F, \varepsilon>0$. Thus $x \in \bar{S}$.

In the next theorem, the centers of orders are related to regular points. The result was first obtained by Dubois [Du, p. 62, Theorem 3].

(1.10) THEOREM. The set of centers of orders of $L$ is the closure of $M(R)_{\mathrm{reg}}$ with respect to the strong topology.

Proof (Suggested by L. Bröcker). Let $x=c(P)$. Take $\varepsilon \in R, \varepsilon>0$. The function $f(y):=\varepsilon-d(x, y)^{2}$ is not zero. If $f \notin P$ then $S=\left\{y \mid d(x, y)^{2}>\right.$ $\varepsilon\} \in \mathscr{F}(P)$, but $x \notin \bar{S}$. Hence $f \in P$ and by (1.3) we find a regular point $y$ with $d(x, y)^{2}<\varepsilon$, i.e. $y \in B(x, \sqrt{\varepsilon})$. This holds for all $\varepsilon$, which means $x$ $\in \overline{M(R)}_{\mathrm{reg}}$. Conversely, if $x \in \overline{M(R)}_{\mathrm{reg}}$ then the open balls $B(x, \varepsilon)$ are open semialgebraic sets which contain a regular point, for $\varepsilon>0$. Hence, they are Zariski-dense by (1.6). Consider the filter $F_{0}$ of semialgebraic subsets of $M(R)$ generated by the open balls $B(x, \varepsilon) . F_{0}$ only contains Zariski-dense subsets. Set $P_{0}=\mathscr{P}\left(F_{0}\right)$ as in 1.7. As in 1.7 we show $P_{0}+P_{0} \subset P_{0}, P_{0} P_{0} \subset P_{0}, P_{0} \cap-P_{0}$ $=\{0\}, L^{2} \subset P_{0}$. It is known, see e.g. [L1], that $P_{0} \subset P$ for some order $P$. One sees $B(x, \varepsilon) \in \mathscr{F}(P)$ for all $\varepsilon$, hence $x=\lim \mathscr{F}(P)$.

The ultrafilter theorem and the notion of a center of an order allow us to interpret orders "geometrically" (at least in certain cases).

ExAmples. (1) $L=\mathbf{R}(X), A=\mathbf{R}[X]$ then $M(\mathbf{R})=\mathbf{R}$. The semialgebraic subsets are finite unions of singletons and open intervals. An ultrafilter $F$ therefore converges to $a \in \mathbf{R}$ or to $-\infty$ or to $+\infty$. Let $\lim F=a$. Consider the function $X-a$. Either $\{X-a>0\} \in F$ or $\{X-a<0\} \in F$.

Let $P=\mathscr{P}(F) ; P$ is determined by $P \cap \mathbf{R}[X]$. In the first case $\{X-a>$ $0\} \in F$, i.e. $X-a \in P$, and $f \in \mathbf{R}[X]$ is in $P$ iff $f \geqslant 0$ on some interval $[a, a+\varepsilon], \varepsilon>0$. We symbolize this order by

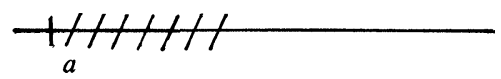

In the other case we have $f \in P$ iff $f \geqslant 0$ on some interval $[a-\varepsilon, a], \varepsilon>0$; of course this is symbolized by

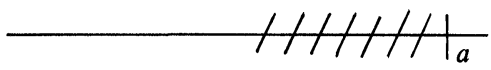

In case $\lim F=+\infty, P \in \mathbf{R}[X]$ is given as the set of $f$ 's which are positive for large values, i.e. have the highest coefficient positive. If $\lim F=-\infty$ then $f \in \mathbf{R}[X] \cap \mathscr{F}(P)$ iff $(-1)^{n} a_{n}>0$ where $n=\operatorname{deg} f, a_{n}$ the highest coefficient.

(2) $L=\mathbf{R}(X, Y), A=\mathbf{R}[X, Y], M(\mathbf{R})=\mathbf{R}^{2}$. In this case there is no such simple description as in (1). Consider for example the semialgebraic partition of $\mathbf{R}^{2}$ by transversal lines through $x$ (Figure 2). There are at least four ultrafilters $F_{i}$ with $S_{i} \in F_{i}$; this means there are orderings $P_{i}$ such that, if $f \geqslant 0$ on $S_{i}$, then $f \in P_{i}$. If we further partition $S_{1}$ into $T_{1}, T_{2}, T_{3}$ as in Figure 3 , then 


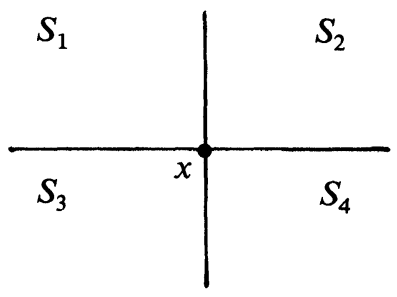

$S_{i}$ open, $i=1,2,3,4$.

FIGURE 2

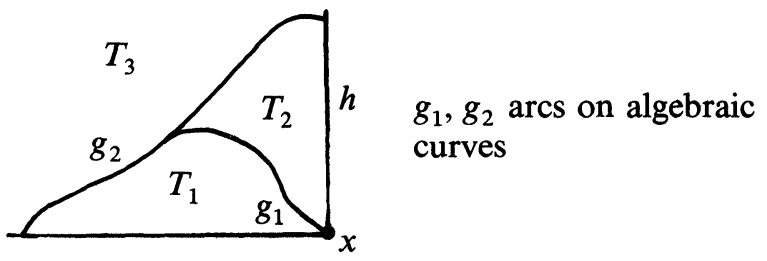

FIGURE 3

we obtain the existence of three further orderings. Thus continuing this process leads to new orderings at each step. If we want $x$ to be the center, then $x$ must lie in all the closures of these semialgebraic sets. In particular, we obtain an ordering $P$ with center $x$ by fixing $h$ and setting $f \in \mathbf{R}[X, Y] \cap P$ iff $f$ is nonnegative on some segment

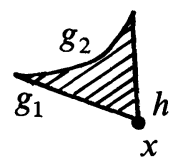

FIGURE 4

on the left upper side of $h$, with corner $x$ and between $h$ and some algebraic curves $g_{1}, g_{2}$ with $g_{1}$ passing through $x$. This order is symbolized by

$$
\text { - } 1 / / / / 1
$$

Of course, the line $h$ may be replaced by any arc of an algebraic curve.

In contrast to the first example, we now do not have any nice interpretation for possible limits of those ultrafilters which do not have a limit in $M(\mathbf{R})$. The situation is even worse if we are dealing with arbitrary real closed fields. In that case, even ultrafilters containing a bounded set need not converge, since $R^{n}$ is not locally compact for $R \neq \mathbf{R}$. However, as we will see in the next section, the real spectrum of $A$ provides, in all cases, a space in which the limits of all ultrafilters $\mathscr{F}(P)$ naturally lie.

So far, we have only studied ultrafilters of Zariski-dense semialgebraic subsets of $M(R)$. But there is no reason not to consider all ultrafilters in 
$\gamma(M(R))$. In the course of this study we will be concerned with subschemes $N=\operatorname{Spec} A / \mathfrak{y}$, where $\mathfrak{y}$ is any prime ideal of $A$. There is the natural embedding $N=\operatorname{Spec} A / \mathfrak{y} \rightarrow M=\operatorname{Spec} A, \mathfrak{g} \mapsto \pi^{-1}(\mathfrak{g}), \pi$ being the natural epimorphism $A \rightarrow A / \mathfrak{y}$, and the Zariski topology on $\operatorname{Spec} A$ induces the Zariski topology on $\operatorname{Spec} A / \mathfrak{y}$. Considering the real points in $N$ and $M$ we have $N(R)=\left\{x \in M(R) \mid f_{1}(x)=\cdots=f_{r}(x)=0\right\}$ where $\mathfrak{y}=\left(f_{1}, \ldots, f_{r}\right)$. Thus, $N(R)$ is considered as an algebraic subset of $M(R)$. Under this identification we identify $\left.f\right|_{N(R)}$ with $f+\mathfrak{y}$ for any $f \in A$. If $\mathfrak{y}$ is assumed to have a formally real residue field $k(\mathfrak{y})=$ quot $(A / \mathfrak{y})$, then by (1.3) and (1.6) $N(R)_{\text {reg }}$ is Zariski-dense in $N$.

EXAMPLE. $A=\mathbf{R}[X, Y], \mathfrak{y}=\left(Y^{2}-X\left(X^{2}-1\right)\right)$. Then $M(\mathbf{R})=\mathbf{R}^{2}, N(\mathbf{R})$ $=\left\{(x, y) \in \mathbf{R}^{2} \mid y^{2}=x\left(x^{2}-1\right)\right\}$.

Now let $F$ be any ultrafilter in $\gamma(M(R))$. We set $\mathfrak{y}(F):=\{f \in A \mid\{f=0\}$ $\in F\}$.

Claim: $\mathfrak{y}=\mathfrak{y}(F)$ is a prime ideal of $A$. That $\mathfrak{y}$ is an ideal is easily seen. Suppose $f g \in \mathfrak{y}$, i.e. $\{f g=0\}=\{f=0\} \cup\{g=0\} \in F$. Since $F$ is an ultrafilter this implies $f \in \mathfrak{y}$ or $g \in \mathfrak{y}$. Thus we have the subscheme $N=\operatorname{Spec} A / \mathfrak{y}$. On $N(R)$ we consider the filter $\left.F\right|_{N(R)}=\{S \in \gamma(N(R)) \mid S \in F\}=: F_{0}$. At least $N(R) \in F_{0}$ since $N(R)=\left\{f_{1}=0\right\} \cap \cdots \cap\left\{f_{r}=0\right\}$ if $\mathfrak{y}=\left(f_{1}, \ldots, f_{r}\right)$. We claim that $F_{0}$ is an ultrafilter of semialgebraic subsets of $N(R)$ which are Zariski-dense in $N$. To see that $F_{0}$ is an ultrafilter in $\gamma(N(R))$ consider $T \in \gamma(N(R))$ satisfying $T \notin F_{0}$. Since $F$ is an ultrafilter we get $M(R) \backslash T \in F$. Using the fact $N(R) \in F$ one derives $N(R) \backslash T=N(R) \cap(M(R) \backslash T) \in F_{0}$ which was to be shown. To see that every set $S$ in $F_{0}$ is Zariski-dense in $N$ we have to show that, given $f \in A$, the statement $f=0$ on $S$ implies $f \in \mathfrak{y}$. Now, $S \subset\{f=0\}$, hence $\{f=0\} \in F$ and $f \in \mathfrak{y}$.

According to the ultrafilter theorem for orders, (1.7), $F_{0}$ induces an order on $k(\mathfrak{y})$. Hence, we assign to every ultrafilter $F$ in $\gamma(M(R))$ a pair $(\mathfrak{y}, \bar{P})$, $\mathfrak{y} \in \operatorname{Spec} A, \bar{P}$ an order on $k(\mathfrak{y})$. This assignment $F \mapsto(\mathfrak{y}, \bar{P})$ is injective since $F=\left\{S \in \gamma(M(R)) \mid S \cap N(R) \in F_{0}\right\}$ and $N(R)$ and $F_{0}$ are determined by $\mathfrak{y}$ and $\bar{P}$.

Conversely, given a pair $(\mathfrak{y}, \bar{P}), \mathfrak{y} \in \operatorname{Spec} A, \bar{P}$ an order on $k(\mathfrak{y})$, we will attach to it an ultrafilter $F$ in $\gamma(M(R))$. The prime ideal $\mathfrak{y}$ provides a subscheme $N=\operatorname{Spec} A / \mathfrak{y}$ and $N(R)$. By (1.7), the order $\bar{P}$ induces an ultrafilter $F_{0}$ in $\gamma(N(R))$. Set $F:=\left\{S \in \gamma(M(R)) \mid S \cap N(R) \in F_{0}\right\}$. Noting $F_{0} \subset F$ one easily checks that $F$ is an ultrafilter in $\gamma(M(R))$ and that the given pair $(\mathfrak{y}, \bar{P})$ is assigned to $F$ by the above construction.

We therefore have that $F \rightarrow(\mathfrak{y}, \bar{P})$ gives a bijection between the set of ultrafilters in $\gamma(M(R))$ and the set of pairs $(\mathfrak{y}, \bar{P})$ where $\mathfrak{y} \in \operatorname{Spec} A, \bar{P}$ an order of $k(\mathfrak{y})$.

Let us now introduce some notations. By $\overline{M(R)}$ we denote the set of all ultrafilters in $\gamma(M(R)) . M(R)$ is naturally embedded in $\widehat{M(R)}$ by attaching to $x \in M(R)$ the principal ultrafilter containing $\{x\}$. We impose on $\widehat{M(R)}$ a topology by taking as a subbasis the sets $\hat{S}=\{F \in \widehat{M(R)} \mid S \in F\}$ where $S=\{f>0\} \subset M(R), f \in A$. In this topology we regard $\overline{M(R)}$ as the ultrafilter completion of $M(R)$, cf. [Br, p. 252]. 
On the other hand we define the real spectrum of an arbitrary ring $A$ by setting

$$
\mathscr{R}-\operatorname{Spec} A=\{(\mathfrak{y}, \bar{P}) \mid \mathfrak{y} \in \operatorname{Spec} A, \bar{P} \text { an order of } k(\mathfrak{y})\}
$$

In the geometric situation, we have, as proved above, a bijection $\widehat{M(R)} \rightarrow \mathscr{R}$ $\operatorname{Spec} A, F \rightarrow(\mathfrak{y}, \bar{P})$. It is readily checked that under this bijection the open set $\hat{S}$ is sent to the set $D(f):=\{(\mathfrak{y}, \bar{P}) \mid f \in A, f+\mathfrak{y} \in \bar{P} \backslash 0\}$. Also, for arbitrary rings, we take the $D(f)$ 's as a subbasis and thus impose a topology on $\mathscr{R}$-Spec $A$.

Summarizing all facts, we have now found the full ultrafilter theorem which first appeared in print in Bröcker's paper [Br2].

(1.11) UltRAFILTER THEOREM. The ultrafilter completion of $M(R)$ is naturally homeomorphic to $\mathscr{R}$-Spec $A$.

The topological space $\mathscr{R}$-Spec $A$ casts new light on the Artin-Lang Theorem (1.3) which already indicated a close link between real regular points and orders of the function field. It is the $\operatorname{space} \mathscr{R}-\operatorname{Spec} A$ which provides a topological description of this relation, as we will see in a moment.

We started with an integral affine algebra $A$ and then passed to its associated affine variety $M=\operatorname{Spec} A$. It is now more appropriate to reverse the order and call $A$ the coordinate ring $R[M]$ of $M$; in this set-up, the quotient field $L$ of $A$ is then the function field $R(M)$ of $M$.

We mentioned the embedding

$$
\Phi_{0}: M(R) \hookrightarrow \operatorname{Spec} R[M], \quad x \mapsto \mathfrak{m}_{x} .
$$

It clearly factors through the embedding

$$
\Phi: M(R) \hookrightarrow \mathscr{R}-\operatorname{Spec} R[M], \quad x \mapsto\left(\mathfrak{m}_{x}, R^{2}\right) .
$$

Whereas $\Phi_{0}$ did not induce the strong topology on $M(R)$, we now have, in the case of $\Phi$ :

(1.12) Proposition. The subspace topology on $M(R)$ inherited from $\mathscr{R}$ $\operatorname{Spec} R[M]$ is just the strong topology.

Proof. The sets $\Phi^{-1}(D(f))=\{x \in M(R) \mid f(x)>0\}$ form a subbasis of the strong topology on $M(R), f \in A$. ding

We have $\mathscr{R}-\operatorname{Spec} R(M)=\{P \mid P$ order of $R(M)\}$ and the canonical embed

$$
\psi: \mathscr{R}-\operatorname{Spec} R(M) \rightarrow \mathscr{R}-\operatorname{Spec} R[M], \quad P \mapsto(\{0\}, P) .
$$

Putting the mappings $\Phi, \psi$ together we obtain the diagram:

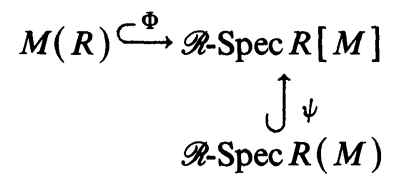


This makes visible how the space $\mathscr{R}$-Spec $R[M]$ combines the two ingredients of the Artin-Lang theorem: the points and the orders. Looking at the images of $\Phi$ and $\psi$ we obtain what may be called the ultimate form of the Artin-Lang theorem (1.3). To simplify the notation we regard $M(R)$ as a subspace of $\mathscr{R}$-Spec $R[M]$ in view of (1.12). Furthermore we set $D\left(f_{1}, \ldots, f_{n}\right)=D\left(f_{1}\right)$ $\cap \cdots \cap D\left(f_{n}\right)$ in $\mathscr{R}-\operatorname{Spec} R[M]$.

(1.13) ARTIN - LANG HomomorPHiSM THEOREM, REVISITED.

(i) $M(R)$ is dense in $\mathscr{R}$-Spec $R[M]$;

(ii) $M(R) \cap \overline{\operatorname{Im} \psi}=\overline{M(R)_{\text {reg }}}$.

REMARK. In (ii), $\overline{M(R)_{\text {reg }}}$ is the closure relative to $M(R)$.

Proof. (i) Given $(\mathfrak{y}, \bar{P}) \in D\left(f_{1}, \ldots, f_{m}\right)$ we have to find $x \in M(R)$ with $f_{i}(x)>0, i=1, \ldots, m$. From the hypothesis, we get $\bar{f}_{i} \in \bar{P}$ in $k(\mathfrak{y})$, where $\bar{f}_{i}=f_{i}+\mathfrak{y}$. By (1.3), there is an $x \in N(R) \subset M(R), N=\operatorname{Spec} A / \mathfrak{y}$, with $f_{i}(x)=f_{i}(x)>0$.

(ii) Take $x \in M(R) \cap \overline{\operatorname{Im} \psi}$ and pick $\varepsilon \in R, \varepsilon>0$. Set $f_{\varepsilon}=\varepsilon-\Sigma\left(\bar{X}_{i}-x_{i}\right)^{2}$ where $x=\left(x_{1}, \ldots, x_{n}\right) \in M(R) \subset R^{n}, \quad \bar{X}_{i}=X_{i}+\mathfrak{a}, \quad R[M]=$ $R\left[X_{1}, \ldots, X_{n}\right] / \mathfrak{a}$. Then $x \in D\left(f_{\varepsilon}\right)$, hence there is an order of $R(M)$ with $f_{\varepsilon} \in P$. By (1.3) we find a regular point $y$ with $f_{\varepsilon}(y)>0$, i.e. $y \in B(x, \sqrt{\varepsilon})$. Hence, $x \in \overline{M(R)_{\text {reg }}}$. Conversely, if $x \in \overline{M(R)_{\text {reg }}}$, then given $f_{1}, \ldots, f_{r}$ with $x \in D\left(f_{1}, \ldots, f_{r}\right)$, i.e. $f_{i}(x)>0$, then, by assumption, there is a regular point $y$ with $f_{i}(y)>0, i=1, \ldots, r$. By (1.3), we find an order $P$ with $f_{1}, \ldots, f_{r} \in P$, i.e. $D\left(f_{1}, \ldots, f_{r}\right) \cap \operatorname{Im} \psi \neq \varnothing$.

2. The topological space $\mathscr{R}-\operatorname{Spec} A$. We start this section by presenting an equivalent definition of the real spectrum of an arbitrary ring $A$. Given $(\mathfrak{y}, \bar{P}) \in \mathscr{R}-\operatorname{Spec} A$ we set $\alpha:=\{a \in A \mid a+\mathfrak{y} \in \bar{P}\}$. Clearly, $\alpha$ satisfies:

$$
\alpha+\alpha \subset \alpha, \quad \alpha \alpha \subset \alpha, \quad \alpha \cup-\alpha=A, \quad \alpha \cap-\alpha \text { is a prime ideal. }
$$

(In this case $\alpha \cap-\alpha=\mathfrak{y}$.) In the general case, every subset $\alpha$ of $A$ satisfying the above conditions is called a prime ordering of $A$ or just an ordering if it is tacitly understood that $\alpha \cap-\alpha$ has to be a prime ideal. Given a prime ordering $\alpha$, the prime ideal $\mathfrak{y}:=\alpha \cap-\alpha$ is called the support of $\alpha, \mathfrak{y}=\operatorname{supp}(\alpha)$. (In the geometric situation of the last section the support $\mathfrak{y}$ describes the subvariety on which the ultrafilter actually lies.) We have the induced prime ordering $\bar{\alpha}=\{a+\mathfrak{y} \mid a \in \alpha\}$ on $A / \mathfrak{y}$ with $\operatorname{supp}(\bar{\alpha})=\{0\}$. Therefore, $\bar{\alpha}$ uniquely extends to an order of $k(\mathfrak{y})=\operatorname{quot}(A / \mathfrak{y})$, again denoted by $\bar{\alpha}$.

We thus have a bijection between $\mathscr{R}$-Spec $A$ and the set of all prime orderings of $A$. In the sequel, we will take this second point of view and will work with prime orderings. Keep in mind that $\alpha$ gives rise to the pair $(\operatorname{supp}(\alpha), \bar{\alpha})$. So, from now on, we write

$$
\mathscr{R} \text {-Spec } A=\{\alpha \mid \alpha \text { prime ordering of } A\} \text {. }
$$

We shall also write "orderings" instead of "prime orderings".

An element $f \in A$ can be viewed as a "function" on $\mathscr{R}$-Spec $A$. We set

$$
f(\alpha):=f+\mathfrak{y} \in k(\mathfrak{y}), \quad \mathfrak{y}=\operatorname{supp}(\alpha) .
$$


By $f(\alpha)>0$ we mean $f(\alpha) \neq 0, f(\alpha) \in \bar{\alpha}$, and by $D(f):=\{f>0\}$ we denote $\{\alpha \in \mathscr{R}$-Spec $A \mid f(\alpha)>0\}$. Note that this present definition is in accordance with the one given before (1.11) if we pass to our new definition of $\mathscr{R}-\operatorname{Spec} A$. Accordingly, we set $\left\{f=0, g_{1}>0, \ldots, g_{r}>0\right\}=\{\alpha \in \mathscr{R}$-Spec $A \mid f(\alpha)=0$, $\left.g_{1}(\alpha)>0, \ldots, g_{r}(\alpha)>0\right\}$. As in the geometric case of the last section, we introduce the lattice with complement which is generated by the sets $\{f>0\}$, $f \in A$. This time the sets obtained are called the constructible subsets of $\mathscr{R}$-Spec $A$. A set is constructible if and only if it is a finite union of sets of the type $\left\{f=0, g_{1}>0, \ldots, g_{r}>0\right\}$. This is readily checked using the fact $\{f=$ $0\} \cap\{g=0\}=\left\{f^{2}+g^{2}=0\right\}$.

The sets $D(f)$ where $f \in A$ constitute by definition a subbasis of a topology on $\mathscr{R}$-Spec $A$. This topology was already defined before (1.11) and is referred to as the topology of $\mathscr{R}-\operatorname{Spec} A$. A basis is given by the sets $D\left(f_{1}, \ldots, f_{r}\right)=$ $\bigcap_{1}^{r} D\left(f_{i}\right), r \in \mathbf{N}, f_{1}, \ldots, f_{r} \in A$. We will also deal with a certain finer topology on $\mathscr{R}$-Spec $A$. This second one is called the Tychonoff topology of $\mathscr{R}$-Spec $A$. It is an auxiliary topology of great importance, at least in proofs.

To introduce the Tychonoff topology, we first note that for any given $\alpha, \beta \in \mathscr{R}-\operatorname{Spec} A$

$$
\alpha \backslash(-\alpha)=\beta \backslash(-\beta) \Rightarrow \alpha=\beta .
$$

Namely, if $f \in \alpha \backslash \beta$ then $-f \in \beta \backslash(-\beta)=\alpha \backslash(-\alpha)$, hence $f \notin \alpha$.

Due to (2.1), we can characterize an ordering $\alpha$ by the characteristic function $e_{\alpha \backslash(-\alpha)}$ of $\alpha \backslash(-\alpha): e_{\alpha \backslash(-\alpha)}(f)=1$ or 0 according to whether or not $f \in \alpha \backslash(-\alpha)$. We consequently get an injective map

$$
\psi:\left\{\begin{aligned}
\mathscr{R}-\operatorname{Spec} A & \rightarrow \prod_{f \in A}\{0,1\}, \\
\alpha & \mapsto\left(e_{\alpha \backslash(-\alpha)}(f)\right) .
\end{aligned}\right.
$$

On $\prod_{f \in A}\{0,1\}$ we consider the product topology yielding, by Tychonoff's theorem, a compact space (compact is to include the Hausdorff property). We claim that the image of $\psi$ is closed in the product topology. By pulling back the topology of $\operatorname{Im} \psi$ to $\mathscr{R}-\operatorname{Spec} A$ via $\psi$, we get what we call the Tychonoff topology of $\mathscr{R}$-Spec $A$, in which $\mathscr{R}$-Spec $A$ is compact. The proof that $\operatorname{Im} \psi$ is closed follows well-known patterns. We therefore only present the typical argument. Assume $x=\left(x_{f}\right) \in \overline{\operatorname{Im} \Phi}$. We have to find $\alpha \in \mathscr{R}-\operatorname{Spec} A$ with $x_{f}=e_{\alpha \backslash(-\alpha)}(f)$. If such an $\alpha$ exists it would coincide with $\left\{f \mid x_{f}=1\right.$ or $\left.x_{f}=x_{-f}=0\right\}$. We therefore study this latter set, call it $\alpha$ and have to show that $\alpha$ satisfies $\alpha+\alpha \subset \alpha, \alpha \alpha \subset \alpha, \alpha \cup-\alpha=A, \alpha \cap-\alpha$ a prime ideal. As an example, we treat the question $\alpha+\alpha \subset \alpha$. Suppose $f, g \in \alpha$ but $f+g \notin \alpha$. The first case to consider might be as follows: $x_{f}=x_{g}=x_{-(f+g)}=1, x_{f+g}=0$. Consider the open neighborhood $U=\left\{\left(y_{h}\right) \in \Pi\{0,1\} \mid y_{f}=1, y_{g}=1, y_{f+g}=\right.$ $0\}$ of $x$. By assumption, there is $\beta \in \mathscr{R}$-Spec $A$, with $\Phi(\beta) \in U$. This means $f, g \in \beta \backslash(-\beta)$ but $f+g \notin \beta \backslash(-\beta)$ which is impossible. All the other cases are treated similarly.

A subbasis of the Tychonoff topology of $\mathscr{R}-\operatorname{Spec} A$ is given by the sets $\{\alpha \mid f \in \alpha \backslash(-\alpha)\}=\{f>0\}$ and $\{\alpha \mid f \notin \alpha \backslash(-\alpha)\}=\{f=0\} \cup\{-f>0\}$. But since $\{f=0\}=\{\alpha \mid f$ and $-f$ not in $\alpha \backslash(-\alpha)\}$ we see that $\{f=0\}$ is also 
open. Note that $\left\{f_{1}=0\right\} \cap \cdots \cap\left\{f_{r}=0\right\}=\left\{\sum_{1}^{r} f_{i}^{2}=0\right\}$. This provides the proof of statement (i) in (2.2); (ii) is clear.

(2.2) Proposition. (i) The constructible subsets of $\mathscr{R}$-Spec $A$ form a basis for the Tychonoff topology of $\mathscr{R}$-Spec $A$.

(ii) In the Tychonoff topology $\mathscr{R}$-Spec $A$ is compact.

(iii) $A$ set is constructible if and only if it is a clopen set (= closed and open set) in the Tychonoff topology.

Proof. (iii) Since the complement of a constructible set is also constructible, these sets are clopen, in view of (i). If a set $S$ is clopen it is, as an open set, a union of constructible sets. A finite number of them already cover $S$ since $S$, being closed, is compact.

As noted, it is not the Tychonoff topology we are really interested in. The topology of $\mathscr{R}$-Spec $A$ has as a basis the sets $D\left(f_{1}, \ldots, f_{r}\right)=\left\{f_{1}>0, \ldots, f_{r}>\right.$ $0\}$. Therefore, it is coarser then the Tychonoff topology. This gives, using (2.2), the

(2.3) TheOREM. $\mathscr{R}$-Spec $A$ is a quasicompact space, every constructible set is also quasicompact.

EXAMPLES. (i) Let $K$ be a formally real field. Then $\mathscr{R}$-Spec $K$ is the set of orders $P$ of $K$, often denoted by $X(K)[\mathbf{B}, \mathbf{L 1}]$. We have $D(f)=\{P \mid f \in P \backslash$ $\{0\}$. In this case, the topology is clearly Hausdorff, rendering $X(K)$ into a compact space. $X(K)$, with its topology, plays an important role in the algebraic theory of quadratic forms [L1]. The topology of $X(K)$ is often referred to as the Harrison topology.

(ii) $A=\mathbf{R}[T]$ : The orderings $\alpha$ with $\operatorname{supp}(\alpha)=0$ are just the sets $P \cap \mathbf{R}[T]$, where $P$ is any order of $\mathbf{R}(T)$. The valuation ring $A(P, \mathbf{R})$ is either $\mathbf{R}\left[T^{-1}\right]_{\left(T^{-1}\right)}$ or $\mathbf{R}[T]_{(T-a)}$ for some $a \in \mathbf{R}$. In the first case we have the two possibilities $P_{\infty}^{ \pm}$defined as follows: It is enough to say when $f \in \mathbf{R}[T]$ lies in $P_{\infty}^{ \pm}$. Let $f=\sum_{0}^{n} a_{i} T^{i}, a_{n} \neq 0$; then $f \in P_{\infty}^{+} \Leftrightarrow a_{n}>0, f \in P_{\infty}^{-} \Leftrightarrow(-1)^{n} a_{n}>0$. In the case of $\mathbf{R}[T]_{(T-a)}$ we also get two possibilities $P_{a}^{ \pm}$. Namely, if $f \in \mathbf{R}[T]$, $f=(T-a)^{k} g(T), g(a) \neq 0$, then $f \in P_{a}^{+} \Leftrightarrow g(a)>0, f \in P_{a}^{-} \Leftrightarrow(-1)^{k} g(a)$ $>0$. Note that the corresponding ultrafilters on the affine line $\mathbf{R}$ have already been dealt with in the examples after (1.10).

Next we consider orderings $\alpha$ with $\mathfrak{y}_{\alpha}=\operatorname{supp}(\alpha) \neq 0$. Then $\mathfrak{y}_{\alpha}=(T-a)$ for some $a \in \mathbf{R}$ and $\alpha=\alpha_{a}=\{f \in \mathbf{R}[T] \mid f(a) \geqslant 0\}, \alpha_{a}$ is associated to the principal ultrafilter containing $\{a\}$, in the map of Ultrafilter Theorem (1.11).

In this case the topology is not Hausdorff. In fact, the singletons are not always closed sets. To prove this consider $P_{a}^{+}$. We claim $\alpha_{a} \in\left\{\overline{P_{a}^{+}}\right\}$. If $\alpha_{a} \in D\left(f_{1}, \ldots, f_{n}\right)$ then we have $f_{i}(a)>0$, which implies $f_{i} \in P_{a}^{+}$. Hence, $\alpha_{a} \in\left\{\overline{P_{a}^{+}}\right\}$. Additionally, one can prove directly $\left\{\overline{P_{a}^{+}}\right\}=\left\{P_{a}^{+}, \alpha_{a}\right\}$; however, this will also follow from more general arguments, see (2.4).

The fact that the topology of $\mathscr{R}$-Spec $A$ is not Hausdorff, even worse, that singletons are not closed in general, might be considered a great disadvantage compared with the nicer Tychonoff topology. However, it is just the structure of the closure of singletons that is the, perhaps, most important feature of 
$\mathscr{R}$-Spec $A$, as we will see in many instances, beginning with the next proposition, where $\alpha, \beta \in \mathscr{R}-\operatorname{Spec} A$.

(2.4) Proposition. $\beta \in \overline{\{\alpha\}} \Leftrightarrow \alpha \subset \beta$.

Proof. $(\Rightarrow)$ Suppose we find $f \in \alpha \backslash \beta$, then $(-f)(\beta)>0$. Hence, $D(-f) \cap$ $\{\alpha\} \neq \varnothing$ which means $(-f)(\alpha)>0, f(\alpha)<0$ but, by assumption, $f(\alpha) \geqslant 0$. $(\Leftarrow)$ Consider a neighborhood $D\left(f_{1}, \ldots, f_{n}\right)$ of $\beta$. We have $f_{i}(\beta)>0$ for all $i$ 's. In case $\alpha \notin D\left(f_{1}, \ldots, f_{n}\right)$ then $f_{i}(\alpha) \leqslant 0$ for some $i$, hence $-f_{i} \in \alpha$, and $-f_{i} \in \beta$ would give the contradiction.

We say that $\alpha, \beta \in \mathscr{R}$-Spec $A$ can be separated if they have mutually disjoint neighborhoods.

(2.5) Proposition. $\alpha, \beta$ can be separated if and only if $\alpha \not \subset \beta, \beta \not \subset \alpha$.

Proof. If $\alpha$ and $\beta$ can be separated then $\alpha \not \subset \beta, \beta \not \subset \alpha$ in view of (2.4). For the converse, pick $f \in \alpha \backslash \beta, g \in \beta \backslash \alpha$ and set $h=f-g$. Then $h(\alpha)=f(\alpha)$ $-g(\alpha)>0, h(\beta)<0$, which gives $\alpha \in D(h), \beta \in D(-h), D(h) \cap D(-h)=$ $\varnothing$.

These last two propositions admit a number of corollaries. To have a convenient terminology we say that $\beta$ specializes $\alpha$ or is a specialization of $\alpha$ if $\alpha \subset \beta$, we write $\alpha \rightarrow \beta$; in this situation, $\alpha$ is called a generalization of $\beta$ or is said to generalize $\beta$. As a first consequence we state

(2.6) Proposition. (i) The specializations of an ordering form a chain under inclusion.

(ii) An ordering is contained in a unique maximal specialization.

(iii) $\{\alpha\}$ is closed if and only if $\alpha$ is a maximal ordering.

Proof. To prove (i) one only observes, by (2.4), that a neighborhood of $\beta$, where $\alpha \rightarrow \beta$, necessarily contains $\alpha$; then (2.5) applies. One easily checks that the union of all specializations of a given $\alpha$ is an ordering, hence the maximal specialization. (iii) follows from (2.4).

The fact that the closure of a singleton $\{\alpha\}$, i.e. the set of all specializations of $\alpha$, is a totally ordered set containing a minimum and a maximum is one of the main features of the topology of $\mathscr{R}-\operatorname{Spec} A$. It has no counterpart in the Zariski spectrum $\operatorname{Spec} A$. M. Knebusch and H. Delfs [Kn, DK] have taken this property as the basis for their choice of the notation "Sper $A$ " for the real spectrum of $A$. The specializations of a given point form a closed chain or, in other words, a spear, and $\mathscr{R}$-Spec $A$ is the union of such spears. Combining this fact with the notation $\operatorname{Spec} A$ for the Zariski-spectrum, they came to introduce the notation Sper $A$. We however, because of the reasons explained in the introduction, will not follow this otherwise nice invention.

The closed points of $\mathscr{R}$-Spec $A$ form a subset which we denote by $\mathscr{R}$-Specm $A$, the maximal real spectrum of $A$, in view of (2.6)(iii). It is a surprising fact that $\mathscr{R}$-Specm $A$ is a compact space, which was first noticed by L. Bröcker. Note that in general $\mathscr{R}$-Specm $A$ is not closed in $\mathscr{R}-\operatorname{Spec} A$.

(2.7) Proposition. $\mathscr{R}$-Specm $A$ is a compact space. 
Proof. That $\mathscr{R}$-Specm $A$ is Hausdorff follows from (2.5). Consider next a cover of $\mathscr{R}$-Specm $A$ by open sets $U_{i}, i \in I$, of $\mathscr{R}-\operatorname{Spec} A$. We claim that the $U_{i}$ 's also form a cover of $\mathscr{R}$-Spec $A$. To see this pick $\alpha$ and let $\beta$ be the maximal specialization of $\alpha$ and be contained in $U_{i}$. Then $\alpha \in U_{i}$ also, because of (2.4). Since $\mathscr{R}$-Spec $A$ is quasicompact, a finite subfamily already covers $\mathscr{R}-\operatorname{Spec} A$, a fortiori $\mathscr{R}$-Specm $A$.

Example. $A=\mathbf{R}[T]$. Here, $\mathscr{R}$-Specm $A=\left\{P_{\infty}^{-}\right\} \cup\left\{\alpha_{a} \mid a \in \mathbf{R}\right\} \cup\left\{P_{\infty}^{+}\right\}$, and is homeomorphic to the compact extended real line $\{-\infty\} \cup \mathbf{R} \cup\{+\infty\}$. This is a really nice fact!

The maximal real spectrum was used by N. Schwartz to characterize abstract affine semialgebraic spaces, see $\$ 4$. As stated in (2.6)(ii) there is a map

$$
\lambda:\left\{\begin{array}{l}
\mathscr{R}-\operatorname{Spec} A \rightarrow \mathscr{R} \text {-Specm } A, \\
\alpha \mapsto \text { maximal specialization of } \alpha .
\end{array}\right.
$$

N. Schwartz [S1] showed that $\lambda$ is continuous. Since $\mathscr{R}$-Spec $A$ is quasicompact this implies that on $\mathscr{R}$-Specm $A$ we have the quotient topology with respect to $\lambda$. To show that $\lambda$ is continuous, one essentially imitates the proof that compact spaces are regular in order to separate a closed point $\alpha$ and a closed set $C \subset \mathscr{R}$-Spec $A$ with $\alpha \notin C$, see [L1] for details.

In the geometric situation, i.e. $A$ an affine algebra over a real closed field, $\mathscr{R}$-Specm $A$ provides the space in which every ultrafilter of semialgebraic sets has a unique limit. The proof of this is postponed to the next section in order to avoid a longer digression.

We now return to the studies of specializations. In particular, we shall see that the term "specialization" is quite appropriate. In fact, every specialization gives rise to certain places between associated fields. For the notion of a place see $[\mathbf{Z S}]$.

Given $\alpha \in \mathscr{R}$-Spec $A$ we have set $\mathfrak{y}_{\alpha}=\operatorname{supp}(\alpha)=\alpha \cap-\alpha$. By $\bar{\alpha}$ we denoted the induced order on $\left.k\left(\mathfrak{y}_{\alpha}\right)=\operatorname{quot}(A / \mathfrak{y})_{\alpha}\right)=A_{\mathfrak{y}_{\alpha}} / \mathfrak{y}_{\alpha} A_{\mathfrak{y}_{\alpha}}$. Now we choose any real closure of $\left(k\left(\mathfrak{y}_{\alpha}\right), \bar{\alpha}\right)$. They are all conjugate over $k\left(\mathfrak{y}_{\alpha}\right)[\mathbf{P C}$. Let $k(\alpha)$ denote a (quite arbitrarily) chosen real closure of $k\left(\mathfrak{y}_{\alpha}\right)$. We will often be in the situation that $k\left(\mathfrak{y}_{\alpha}\right)$ lies inside some real closed field $R$ which induces the order $\bar{\alpha}$ on $k\left(\mathfrak{y}_{\alpha}\right)$, i.e. $\bar{\alpha}=k\left(\mathfrak{y}_{\alpha}\right) \cap R^{2}$. In this case, $R$ contains a unique real closure, namely the algebraic closure of $k\left(\mathfrak{y}_{\alpha}\right)$ in $R$, which is then to be taken as $k(\alpha)$.

There is always the natural map

$$
\pi_{\alpha}: A \rightarrow A / \mathfrak{y}_{\alpha} \rightarrow k(\alpha), \quad a \mapsto a+\mathfrak{y}_{\alpha} \in k(\alpha),
$$

and we have $\pi_{\alpha}^{-1}\left(k(\alpha)^{2}\right)=\alpha$. Therefore, the orderings of $A$ are just the preimages of the positive cones of real closed fields $R$ under all possible homomorphisms $A \rightarrow R$. Suppose first that $\mathfrak{y}_{\alpha} \subset \mathfrak{y}_{\beta}$ for $\alpha, \beta \in \mathscr{R}$-Spec $A$. We then have the homomorphism $\mu_{\alpha, \beta}: A / \mathfrak{y}_{\alpha} \rightarrow k(\beta), a+\mathfrak{y}_{\alpha} \mapsto a+\mathfrak{y}_{\beta} \in k(\beta)$. Keeping in mind the orderings $\bar{\alpha}, \bar{\beta}$ on $A / \mathfrak{y}_{\alpha}, A / \mathfrak{y}_{\beta}$ respectively we immediately have:

(2.8) Proposition. The following statements are equivalent:

(i) $\alpha \subset \beta$.

(ii) $\mathfrak{y}_{\alpha} \subset \mathfrak{y}_{\beta}$ and $\mu_{\alpha, \beta}$ is order preserving. 
We now assume $\alpha \subset \beta$. Then $\mu_{\alpha, \beta}: A / \mathfrak{y}_{\alpha} \rightarrow k(\beta)$ is order preserving with $\operatorname{ker} \mu_{\alpha, \beta}=\mathfrak{y}_{\beta} / \mathfrak{y}_{\alpha}$. Writing $\mu$ for $\mu_{\alpha, \beta}$, we set $A_{\alpha, \beta}=\left(A / \mathfrak{y}_{\alpha}\right)_{\operatorname{ker} \mu}$. The ring $A_{\alpha, \beta}$ is a local subring of $k(\alpha)$, in fact of $k\left(\mathfrak{y}_{\alpha}\right)$, and $\mu$ uniquely extends to a homomorphism $\mu: A_{\alpha, \beta} \rightarrow k\left(\mathfrak{y}_{\beta}\right)$ which again is order preserving with respect to the orders $\bar{\alpha}, \bar{\beta}$ of $k(\alpha)$ and $k(\beta)$ respectively. Hence, the maximal ideal of $A_{\alpha, \beta}$, being the kernel of $\mu$, is convex in $A_{\alpha, \beta}$. Now the place extension theorem of Brumfiel, in the form given in (1.8), applies. We get the existence of a place $\tilde{\mu}: k(\alpha) \rightarrow K \cup \infty$, yielding a commutative diagram:

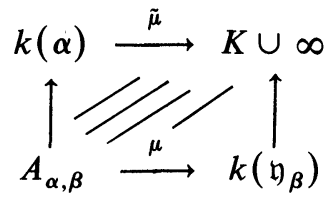

We may assume that $K$ is the residue field of $k(\alpha)$ under $\tilde{\mu}$. Then $K$ is itself real closed, see [PC]. Thus we have proved half of the following result:

(2.9) Proposition. The following statements are equivalent:

(i) $\alpha \subset \beta$.

(ii) $\mathfrak{y}_{\alpha} \subset \mathfrak{y}_{\beta}, \mu_{\alpha, \beta}: A / \mathfrak{y}_{\alpha} \rightarrow A / \mathfrak{y}_{\beta}$ extends to a place $\tilde{\mu}: k(\alpha) \rightarrow K \cup \infty$, where $K$ is a real closed extension of $\left(k\left(\mathfrak{y}_{\beta}\right), \bar{\beta}\right)$.

Proof. Only the proof of (ii) $\Rightarrow$ (i) is missing. Since a positive element in $k(\alpha)$ is a square and any valuation ring is integrally closed we get that $\tilde{\mu}$, and hence $\mu$, is order preserving . Now (2.8) applies.

In general there are many extensions of $\mu_{\alpha, \beta}$ to places on $k(\alpha)$. In the fourth section, we shall deal with the set of all these extensions.

We next consider the topology of subspaces $Y$ of $\mathscr{R}$-Spec $A$. To simplify matters we assume that $Y$ is closed in the Tychonoff topology or, as can easily be checked, that $Y$ is an intersection of constructible subsets of $\mathscr{R}$-Spec $A$. Thus, in particular, all our results are applicable to constructible sets.

Given $\alpha \in Y$, we will first look at the specialization of $\alpha$ in $Y$. Here we have:

(2.10) Proposition. (i) $\alpha$ admits a unique maximal specialization in $Y$,

(ii) $\alpha$ is maximal in $Y$ iff $\{\alpha\}$ is closed in $Y$,

(iii) $Y^{\max }:=\{\alpha \in Y \mid \alpha$ maximal in $Y\}$ is a compact space,

(iv) the map $Y \rightarrow Y^{\max }, \alpha \mapsto$ maximal specialization of $\alpha$, is continuous.

Proof. Once (i) is proved one can argue as previously. Consider $\gamma=\bigcup \beta$ where $\alpha \subset \beta, \beta \in Y$. As the set of these $\beta$ 's is totally ordered under inclusion, we have that $\gamma$ is an ordering of $A$. Suppose $\gamma \notin Y$. Then, by the definition of the Tychonoff topology, there is a constructible set $S=\left\{f=0, g_{1}>0, \ldots, g_{r}\right.$ $>0\}$ containing $\gamma$ but itself being contained in the complement of $Y$. Now $f(\gamma)=0$ implies that $f,-f \in \gamma$ hence $f,-f \in \beta$, i.e. $f(\beta)=0$, for some $\beta \supset \alpha$, $\beta \in Y$. Then $\gamma \in \overline{\{\beta\}}$ by (2.4), and $g_{i}(\gamma)>0$ imply $g_{i}(\beta)>0$, thus $\beta \in S$ : a contradiction.

As we did in the case of $\mathscr{R}$-Spec $A$, we may now also rephrase (i) by stating that $Y$ is a "union of spears". 
Note that $Y$ also carries the subspace Tychonoff topology. Closed, respectively open, subsets of $Y$ are referred to as Tychonoff- closed, respectively Tychonoff-open, if this topology is being considered.

(2.11) Proposition. A subset $X \subset Y$ is closed (resp. open) in $Y$ iff it is Tychonoff-closed (resp. Tychonoff-open) and is closed with respect to specializations (resp. generalizations) in $Y$.

Proof. It is enough to consider the characterization of closed sets, and in this case we only show that $X$ is closed provided the other properties are assumed. Let $\beta \in \bar{X} \cap Y$, then $X \cap D\left(f_{1}, \ldots, f_{r}\right) \neq \varnothing$ for any given $f_{1}, \ldots, f_{r}$ with $f_{i}(\beta)>0$. Now, in the Tychonoff topology of $\mathscr{R}$-Spec $A$, the sets $X \cap$ $D\left(f_{1}, \ldots, f_{r}\right)$ are nonempty closed subsets of the compact space $X$. Therefore, their intersection is not empty. This means we can find $\alpha \in X$ with $f(\alpha)>0$, whenever $f(\beta)>0$. But, by (2.4), this implies $\alpha \subset \beta, \alpha \in X, \beta \in Y$ and, by assumption, $\beta \in X$.

In this relative situation, we also introduce the notion of sets that are "constructible in $Y$ ". By definition, these are sets $Y \cap C$, where $C$ is any constructible set in $\mathscr{R}-\operatorname{Spec} A$. They are clopen in the Tychonoff topology of $Y$ of which they form a basis. They are quasicompact subsets of $Y$, but in general they are neither open nor closed. It is clear that finite unions of sets of the type $Y \cap D\left(f_{1}, \ldots, f_{r}\right)$ are open and constructible relative to $Y$ : these sets are referred to as open-constructible. Equally, the finite unions of the type $Y \cap\left\{f_{1} \geqslant 0, \ldots, f_{r} \geqslant 0\right\}$ are closed and constructible in $Y$ and are referred to as closed-constructible. The next result is easily proved by keeping in mind that a set constructible in $Y$ is quasicompact and that the open-constructibles in $Y$ form a basis of the topology. However, despite its simplicity, it will finally lead to the so-called finiteness theorem of semialgebraic geometry in the next section.

(2.12) Proposition. Let $X$ be constructible in $Y$, then $X$ is open (resp. closed) in $Y$ iff $X$ is open-constructible (resp. closed-constructible) in $Y$.

We will conclude this section by looking at $\mathscr{R}-\operatorname{Spec} A$ as a functor on the category of commutative rings with unit into the category of topological spaces. Given a homomorphism $\varphi: A \rightarrow B, A, B$ rings, we obtain the induced map

$$
\varphi_{*}=\left\{\begin{aligned}
\mathscr{R}-\operatorname{Spec} B & \rightarrow \mathscr{R} \text {-Spec } A, \\
\beta & \mapsto \varphi^{-1}(\beta) .
\end{aligned}\right.
$$

It is easily checked that $\varphi_{*}(\beta)=\varphi^{-1}(\beta)$ is indeed an ordering of $A$. Setting $\alpha=\varphi^{-1}(\beta)$ we get $\operatorname{supp}(\alpha)=\varphi^{-1} \operatorname{supp}(\beta)$. Therefore, we obtain the orderpreserving monomorphism

$$
\left(A / \mathfrak{y}_{\alpha}, \bar{\alpha}\right) \stackrel{\bar{\varphi}}{\rightarrow}\left(B / \mathfrak{y}_{\beta}, \bar{\beta}\right), \quad a+\mathfrak{y}_{\alpha} \mapsto \varphi(a)+\mathfrak{y}_{\beta} .
$$

If $f \in A$ then

$$
\bar{\varphi}\left[f\left(\varphi_{*}(\beta)\right)\right]=\varphi(f)(\beta) .
$$


Consequently, $\varphi_{*}^{-1}(D(f))=D(\varphi(f))$, and this leads to

(2.14) Proposition. $\varphi_{*}$ is continuous in the topologies of real spectra and in the Tychonoff topologies, and the inverse images of constructible sets are constructible sets.

We have $(\varphi \psi)_{*}=\psi_{*} \varphi_{*}$, thus the last result may be rephrased by stating that $A \rightarrow \mathscr{R}$-Spec $A$ is indeed a contravariant functor.

The direct image $\varphi_{*}(C)$ of a constructible $C$ is in general no longer constructible. All that can be said in general is that $\varphi_{*}(C)$ is an intersection of constructible sets, since $\varphi_{*}(C)$ is, in the Tychonoff topology, an image of a compact set under a continuous map, hence closed in the Tychonoff topology. To obtain an example where $\varphi_{*}(C)$ is not constructible, consider an $R$-affine integral domain $A, R$ a real closed field, with $\operatorname{dim} A \geqslant 1$ and function field $F$. The inclusion $i: A \rightarrow F$ gives rise to $i_{*}: \mathscr{R}-\operatorname{Spec} F \rightarrow \mathscr{R}-\operatorname{Spec} A$. Assume the image of $i_{*}$ is constructible, i.e. $i_{*}(\mathscr{R}-\operatorname{Spec} F)=\{A \cap P \mid P \operatorname{order} F\}$ is a finite union of nonempty sets of the type

$$
D\left(f ; g_{1}, \ldots, g_{r}\right)=\left\{\alpha \mid f(\alpha)=0, g_{1}(\alpha)>0, \ldots, g_{r}(\alpha)>0\right\} .
$$

Since $f(A \cap P)=f$ we can eliminate all $f$ 's. But then (1.3) guarantees the existence of a maximal ideal $\mathfrak{m}$ of $A$ with $A / \mathfrak{m}=R$ and $g_{i}+\mathfrak{m}>0$, $i=1, \ldots, r$. Hence, we obtain $\alpha:=\{h \in A \mid h+\mathfrak{m} \geqslant 0\}$ in $D\left(g_{1}, \ldots, g_{r}\right)$, which is evidently not of the form $A \cap P, P$ an order of $F$.

However, there are cases where the image $\varphi_{*}(C)$ is again constructible. In [CC1, 2.3], Coste and Coste-Roy proved

(2.15) THEOREM. If $\varphi: A \rightarrow B$ makes $B$ into a finitely presented $A$-algebra then $\varphi_{*}(C)$ is constructible for any constructible subset $C$ of $\mathscr{R}$-Spec $B$.

In the sequel we shall give a proof which is essentially their proof, based on model theory. The proof will illustrate the fact that $(2.15)$ is a simple consequence of the elimination of quantifiers in the theory of real closed fields. Moreover, a refusal to use this fundamental model-theoretic result would even prevent clear insight into what is really the crucial point when dealing with the question whether $\varphi_{*}$ preserves constructibility or not. In analyzing what to do in order to prove (2.15) we at once meet the problem of studying the fibers of $\varphi_{*}$. Indeed, if $\varphi: A \rightarrow B$ and $C$, constructible in $\mathscr{R}-\operatorname{Spec} B$, are given, then

$$
\varphi_{*}(C)=\left\{\alpha \in \mathscr{R}-\operatorname{Spec} A \mid \varphi_{*}^{-1}(\alpha) \cap C \neq \varnothing\right\} .
$$

For convenience we recall our convention that we have always chosen a fixed real closure $k(\alpha)$ for an ordering $\alpha$ of a ring $A$, i.e. we attach to $\alpha$ a fixed homomorphism $\pi_{\alpha}: A \rightarrow k(\alpha)$, where $\alpha=\left(\pi_{\alpha}\right)_{*}\left(k(\alpha)^{2}\right)$ and $k(\alpha)$ is the chosen real closure of $\left(k\left(\mathfrak{y}_{\alpha}\right), \bar{\alpha}\right)$.

(2.16) Proposition. Let $A \stackrel{\varphi}{\rightarrow} B \stackrel{\psi}{\rightarrow} C$ be ring monomorphisms and $\alpha, \beta, \gamma$ orders of $A, B, C$ respectively. Then the following statements hold: 
(i) If $\varphi_{*}(\beta)=\alpha$ then there exists a unique homomorphism $\bar{\varphi}: k(\alpha) \rightarrow k(\beta)$ yielding a commutative diagram:

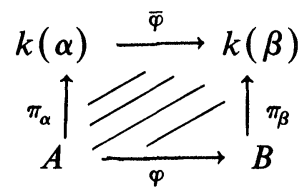

(ii) if $\psi_{*}(\gamma)=\beta, \varphi_{*}(\beta)=\alpha$ then $\bar{\psi} \circ \bar{\varphi}=\overline{\psi \circ \varphi}$.

Proof. As follows from the arguments before (2.13), $\varphi$ induces an orderpreserving monomorphism $\bar{\varphi}:\left(k\left(\mathfrak{y}_{\alpha}\right), \bar{\alpha}\right) \rightarrow\left(k\left(\mathfrak{y}_{\beta}\right), \bar{\beta}\right)$. It now follows from the theory of real closed fields [PC, p. 32] that $\bar{\varphi}$ uniquely extends to a homomorphism $\bar{\varphi}: k(\alpha) \rightarrow k(\beta)$.

To prove (ii) we need only show that $\bar{\psi} \circ \bar{\varphi}$ and $\overline{\psi \circ \varphi}$ coincide on $\pi_{\alpha} A$, since they are then equal on $k\left(\mathfrak{y}_{\alpha}\right)$ and, as extensions are unique, we get $\bar{\psi} \circ \bar{\varphi}$ $=\overline{\psi \circ \varphi}$. But $\bar{\psi} \circ \bar{\varphi} \circ \pi_{\alpha}=\overline{\psi \circ \varphi} \circ \pi_{\alpha}$ follows from the diagram in (i).

(2.17) THEOREM [CC1, 4.3]. If $\alpha \in \mathscr{R}-\operatorname{Spec} A$ and $A \stackrel{\varphi}{\rightarrow} B$ is a ring homomorphism then the canonical map $i: B \rightarrow B \otimes_{A} k(\alpha)$ induces a homeomorphism $i_{*}$ : $\mathscr{R}-\operatorname{Spec}\left(B \otimes_{A} k(\alpha)\right) \rightarrow \varphi_{*}^{-1}(\alpha)$.

Proof. We consider $B$ and $k(\alpha)$ as $A$-algebras induced by the homomorphism $\varphi$ and $\pi_{\alpha}$ respectively. In this way we get the commutative diagram:

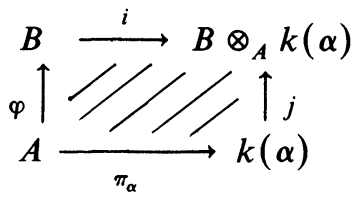

$$
\begin{aligned}
& i(b)=b \otimes_{A} 1, \\
& j(c)=1 \otimes_{A} c .
\end{aligned}
$$

Since $i \varphi=j \pi_{\alpha}$ and $j_{*}(\gamma)=k(\alpha)^{2}$, for any $\gamma \in \mathscr{R}$-Spec $B \otimes_{A} k(\alpha)$, we see that $\varphi_{*}\left(i_{*}(\gamma)\right)=\left(\pi_{\alpha}\right)_{*} k(\alpha)^{2}=\alpha$ for any such $\gamma$. Thus $\operatorname{Im} i_{*} \subset \varphi_{*}^{-1}(\alpha)$. To prove surjectivity, take $\beta \in \mathscr{R}$-Spec $B$ with $\varphi_{*}(\beta)=\alpha$. By (2.16)(i), we have $\bar{\varphi}$ : $k(\alpha) \rightarrow k(\beta)$ with $\bar{\varphi} \pi_{\alpha}=\pi_{\beta} \varphi$. Therefore, we can define a ring homomorphism $\pi: B \otimes_{A} k(\alpha) \rightarrow k(\beta)$ by setting $\pi\left(b \otimes_{A} c\right)=\pi_{\beta}(b) \bar{\varphi}(c)$. Clearly, $\pi i=\pi_{\beta}$, hence $i_{*}\left(\pi_{*} k(\beta)^{2}\right)=\beta$. Injectivity is more complicated. We first show: if $i_{*}(\gamma)=\beta$ then $i: k(\beta) \rightarrow k(\gamma)$ is an isomorphism. Consider the diagram:

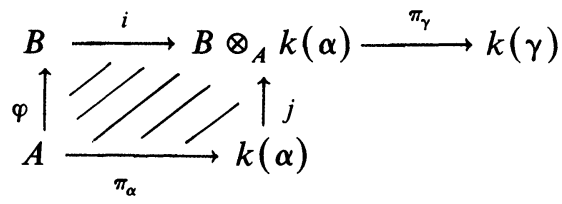

By definition, $k(\gamma)$ is the real closure of the quotient field $F$ of the image of $\pi_{\gamma}$. If we can show $F \subset \bar{i} k(\beta) \subset k(\gamma)$ then $\bar{i}(k(\beta))=k(\gamma)$ must follow since $\bar{i}(k(\beta))$ is real closed. Now, as a ring, $\operatorname{Im}\left(\pi_{\gamma}\right)$ is generated by $\pi_{\gamma} i(B)$ and $\pi_{\gamma} j(k(\alpha))$. Clearly $\pi_{\gamma} i(B)=\bar{i} \pi_{\beta}(B) \subset \bar{i}(k(\beta))$. To determine the image of $\pi_{\gamma} j$ we appeal to (2.16); from (i) we get $\pi_{\gamma} j=\bar{j}$ and from (ii) $\bar{i} \bar{\varphi}=\bar{j}$ follows. Hence, $\operatorname{Im}\left(\pi_{\gamma} j\right) \subset \operatorname{Im} \bar{i}$, and the claim, that $\bar{i}$ is an isomorphism, is proved. 
Next assume $i_{*}(\gamma)=i_{*}(\delta)=\beta$. Since $\bar{i}_{\gamma}: k(\beta) \rightarrow k(\gamma)$ and $\bar{i}_{\delta}: k(\beta) \rightarrow$ $k(\delta)$ are isomorphisms we get an isomorphism $\psi: k(\gamma) \rightarrow k(\delta)$ with $\psi \bar{i}_{\gamma}=\bar{i}_{\delta}$. If we can show $\psi \pi_{\gamma}=\pi_{\delta}$ then evidently $\gamma=\delta$. Since $\pi_{\gamma}\left(b \otimes_{A} c\right)=$ $\left[\left(\pi_{\gamma} i\right)(b)\right]\left[\left(\pi_{\gamma} j\right)(c)\right]$ holds (and the same for $\delta$ ) it is enough to show $\psi \pi_{\gamma} i=\pi_{\delta} i$, $\psi \pi_{\gamma} j=\pi_{\delta} j$. We get

$$
\begin{aligned}
& \psi \pi_{\gamma} i=\psi\left(\bar{i}_{\gamma} \pi_{\beta}\right)=\left(\psi \bar{i}_{\gamma}\right) \pi_{\beta}=\bar{i}_{\delta} \pi_{\beta}=\pi_{\delta} i \\
& \psi \pi_{\gamma} j=\psi \bar{j}_{\gamma}=\psi\left(\bar{i}_{\gamma} \bar{\varphi}\right)=\left(\psi \bar{i}_{\gamma}\right) \bar{\varphi}=\bar{i}_{\delta} \bar{\varphi}=\bar{j}_{\delta}=\pi_{\delta} j .
\end{aligned}
$$

Thus, $i_{*}: \mathscr{R}-\operatorname{Spec}\left(B \otimes_{A} k(\alpha)\right) \rightarrow \varphi_{*}^{-1}(\alpha)$ is shown to be a bijection. It is left to prove that $i_{*}$ is homeomorphic. Since $i_{*}$ is continuous by (2.14), we thus have to prove that $i_{*}$ is an open map. In order to do this, we appeal to (2.11) since $Y:=\varphi_{*}^{-1}(\alpha)$ is closed in the Tychonoff topology of $\mathscr{R}-\operatorname{Spec} B$ ( $\varphi_{*}$ is continuous, $\{\alpha\}$ is closed in this topology). Note that $i_{*}$ is a homeomorphism in the Tychonoff topology since it is continuous and all spaces in question are compact. It is sufficient to show that sets of the form $i_{*}(D(f))$ are open in $\varphi_{*}^{-1}(\alpha)$. Thus, assume $\beta, \gamma \in \varphi_{*}^{-1}(\alpha), \beta \subset \gamma, \gamma \in i_{*}(D(f))$. We have to show by $(2.11)$ that $\beta \in i_{*}(D(f))$. Now

$$
\beta=i_{*}(\delta), \quad \gamma=i_{*}(\varepsilon) \text { for } \delta, \varepsilon \in \mathscr{R}-\operatorname{Spec}\left(B \otimes_{A} k(\alpha)\right)
$$

and, for simplicity, we may assume $k(\beta)=k(\delta), k(\gamma)=k(\varepsilon)$. Now, since $\gamma$ specializes $\beta$ there is, according to (2.9), a place $\lambda: k(\beta) \rightarrow K \cup \infty$ with $K$ real closed, $k(\gamma) \subset K, \lambda \pi_{\beta}=\pi_{\gamma}$. The homomorphism $A \stackrel{\varphi}{\rightarrow} B$ induces $k(\alpha) \stackrel{\bar{\phi}_{\beta}}{\rightarrow} k(\beta)$ and $k(\alpha) \stackrel{\bar{\varphi}_{\gamma}}{\rightarrow} k(\gamma)$. We next show that $\lambda$ is finite on $\bar{\varphi}_{\beta}(k(\alpha))$ and that $\lambda \bar{\varphi}_{\beta}=\bar{\varphi}_{\gamma}$ holds. In the diagram

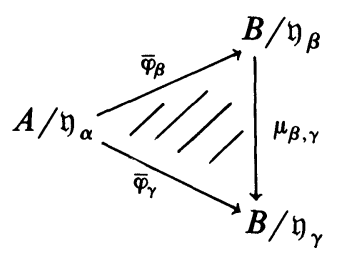

we see that $\bar{\varphi}_{\beta}^{-1}\left(\operatorname{ker} \mu_{\beta, \gamma}\right)=0$ since $\bar{\varphi}^{-1}\left(\mathfrak{y}_{\beta}\right)=\bar{\varphi}^{-1}\left(\mathfrak{y}_{\gamma}\right)=\mathfrak{y}_{\alpha}$. Hence we obtain an extension

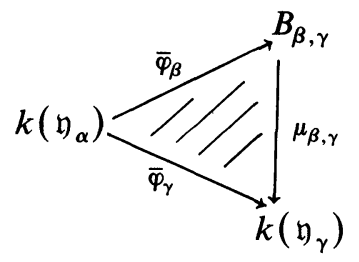

where all maps are order-preserving. Since $B_{\beta, \gamma} \subset V_{\lambda}, V_{\lambda}$ being the valuation ring of $\lambda$, we see that the real closure of $\bar{\varphi}_{\beta}\left(k\left(\mathfrak{y}_{\alpha}\right)\right)$, i.e. $\bar{\varphi}_{\beta}(k(\alpha))$, is contained in $V_{\lambda}\left(V_{\lambda}\right.$ is integrally closed). Moreover, $\lambda \circ \bar{\varphi}_{\beta}=\mu_{\beta, \gamma} \circ \bar{\varphi}_{\beta}=\bar{\varphi}_{\gamma}$ on $k\left(\mathfrak{y}_{\alpha}\right)$ and the uniqueness of extension yields $\lambda \circ \bar{\varphi}_{\beta}=\bar{\varphi}_{\gamma}$ on $k(\alpha)$. We can now prove 
$\beta \in i_{*}(D(f))$. Set $f=\sum b_{l} \otimes_{A} a_{l}$. Then $\gamma \in i_{*}(D(f))$ means $\gamma=i_{*}(\varepsilon), \pi_{\varepsilon}(f)$ $>0$. We have $\pi_{\delta}(f)=\Sigma\left(\pi_{\delta} i\right)\left(b_{l}\right)\left(\pi_{\delta} j\right)\left(a_{l}\right)$ and since $\pi_{\delta} i=\pi_{\beta}, \pi_{\delta} j=\bar{\varphi}_{\beta}$ (note $\bar{i}=\mathrm{id})$, we obtain $\pi_{\delta}(f) \in V_{\lambda}$ and then, in view of $\lambda \pi_{\beta}=\pi_{\gamma}$ and $\lambda \bar{\varphi}_{\beta}=\bar{\varphi}_{\gamma}$, this leads to $\lambda \pi_{\delta}(f)=\pi_{\varepsilon}(f)$ which forces $\pi_{\delta}(f)>0$ and $\beta \in i_{*} D(f)$.

This last theorem is a result that really deserves attention: surprisingly, it comprises several seemingly different aspects. Let us consider two of these. First assume that $K$ is an ordered field with real closure $R$ and $L$ a finite extension. Now $L \otimes_{K} R$ is a product of several copies (if any) of $R$ and $R(\sqrt{-1})$. We thus recover the well-known fact that the extensions of the order of $K$ uniquely correspond to the $K$-embeddings of $L$ into $R$. As a second example, let $K, R$ be as above and consider all extensions of the order of $K$ to the rational function field $K\left(T_{1}, \ldots, T_{n}\right)$. Clearly, every order of $R\left(T_{1}, \ldots, T_{n}\right)$ restricts to an extension on $K\left(T_{1}, \ldots, T_{n}\right)$. Now, (2.17) tells us that this restriction induces a homeomorphism between the space of all extensions to $K\left(T_{1}, \ldots, T_{n}\right)$ and the order space of $R\left(T_{1}, \ldots, T_{n}\right)$.

It is also worth noting why Theorem (2.17) is true. To this end let $A=k$ denote an ordered field with the order $\alpha$. Set $B=k[T]$ and $R$ a real closure of $(k, \alpha)$. Then $(2.17)$ states that $\mathscr{R}$-Spec $R[T] \stackrel{i_{*}}{\rightarrow} \mathscr{R}$-Spec $k[T]$ is injective. This can be seen directly in the following way: if $(T-a) \cap k[T]=(T-b) \cap k[T]$ for $a, b \in R$ then $a$ and $b$ are $k$-conjugate inside $R$. This enforces $a=b$ since otherwise we would find a nontrivial $k$-automorphism of $R$, contradicting $\operatorname{Aut}(R \mid k)=1$. It is this rigidity of $R \mid k$, i.e. $\operatorname{Aut}(R \mid k)=1$, which lies on the basis of (2.17) as L. Bröcker has pointed out to me. If one namely replaces the real closure by the algebraic closure $K$ and the real spectrum by the Zariskispectrum then the canonical map Spec $K[T] \rightarrow \operatorname{Spec} k[T]$ is no longer injective since for $k$-conjugate elements $a, b \in R$ we clearly have $(T-a) K[T] \cap k[T]$ $=(T-b) K[T] \cap k[T]$.

We are now ready to prove (2.15). Without loss of generality we may assume $B=A\left[T_{1}, \ldots, T_{n}\right] /\left(F_{1}, \ldots, F_{m}\right)$ and that $\varphi=\pi \circ j$, where $j: A \rightarrow A\left[T_{1}, \ldots, T_{n}\right]$ and $\pi: A\left[T_{1}, \ldots, T_{n}\right] \rightarrow B$ are the natural morphisms. Now, $\pi_{*}(C)$ is easily seen to be constructible provided $C$ is constructible. Thus we really have to deal with the situation $\varphi: A \rightarrow A\left[T_{1}, \ldots, T_{n}\right], \varphi$ the inclusion map. It is furthermore sufficient to assume that $C=\left\{F=0, G_{1}>0, \ldots, G_{r}>0\right\}$. Now under $i: A\left[T_{1}, \ldots, T_{n}\right] \rightarrow k(\alpha)\left[T_{1}, \ldots, T_{n}\right]$, we have that $\varphi_{*}^{-1}(\alpha) \cap C$ corresponds to the constructible set $\bar{C}=\left\{\bar{F}=0, \bar{G}_{1}>0, \ldots, \bar{G}_{t}>0\right\}$, where $\bar{F}$ denotes $i(F)$ etc., i.e. $\bar{F}$ is the polynomial over $k(\alpha)$ obtained after reducing the coefficients modulo $\mathfrak{y}_{\alpha}$. In the next section we will prove in (3.2) that $\bar{C} \neq \varnothing$ if and only if there is a point $x=\left(x_{1}, \ldots, x_{n}\right) \in k(\alpha)^{n}$ with $\bar{F}(x)=0$, $\bar{G}_{1}(x)>0, \ldots, \bar{G}_{r}(x)>0$. (The proof is postponed at this point to avoid digression.) What we now have to do is to express the existence of such a solution $x \in k(\alpha)^{n}$ of the system $\bar{F}=0, \bar{G}_{1}>0, \ldots, \bar{G}_{r}>0$ by a finite boolean combination of statements of the form $H\left(\bar{a}_{1}, \bar{a}_{2}, \ldots\right)>0$ where $H$ is a polynomial over $\mathbf{Z}$ and the $\bar{a}_{1}, \bar{a}_{2}, \ldots$ are the images of the coefficients $a_{1}, a_{2}, \ldots$ of $F, G_{1}, \ldots, G_{r} \in A\left[T_{1}, \ldots, T_{n}\right]$ in $k(\alpha)$. But exactly this is provided by Tarski's theorem of the elimination of quantifiers in the theory of real closed fields. By considering the elements $H\left(a_{1}, a_{2}, \ldots\right)$ in $A$ for every 
occurring polynomial $H$, we get, according to the above boolean combination, a constructible set $S$ in $\mathscr{R}$-Spec $A$ such that $\varphi_{*}^{-1}(\alpha) \cap C \neq \varnothing$ if and only if $\alpha \in S$. Therefore, $\varphi_{*}(C)$ is constructible. For model theory cf. [P, $\left.\mathbf{S}\right]$.

3. First applications to semialgebraic geometry. In this section we resume the considerations of $\S 1$. Thus, let $R$ be a real closed field and $A$ an affine integral domain over $R$ with associated affine algebraic set $M(R)$. We also use the notation $R[M]=A$ and $F$ for the quotient field of $A$. Recall from $\S 1$ the embedding

$$
\Phi:\left\{\begin{aligned}
M(R) & \hookrightarrow \mathscr{R}-\operatorname{Spec} R[M], \\
x & \mapsto \alpha_{x}=\{f \mid f(x) \geqslant 0\} .
\end{aligned}\right.
$$

In $\S 1$ we wrote $\Phi(x)=\left(\mathfrak{m}_{x}, R^{2}\right)$ which clearly corresponds to the ordering $\alpha_{x}$. We therefore write $\Phi(x)=\alpha_{x}$.

If $C$ is a constructible set then $C \cap M(R):=\Phi^{-1}(C)$ is a semialgebraic set and, conversely, every semialgebraic set in $M(R)$ is of the form $C \cap M(R)$ for some constructible set $C$ in $\mathscr{R}$-Spec $R[M]$. To see this, one only needs to note that $\left\{f=0, g_{1}>0, \ldots, g_{r}>0\right\} \cap M(R)=\left\{x \in M(R) \mid f(x)=0, g_{1}(x)>\right.$ $\left.0, \ldots, g_{r}(x)>0\right\}$ holds. It will turn out in a moment that $C \mapsto C \cap M(R)$ is, in fact, a one-to-one correspondence between constructible sets and semialgebraic sets. But before proving this we want to exploit the observation that the above map is actually a map

$$
M(R) \rightarrow \mathscr{R} \text {-Specm } R[M] .
$$

By (1.13), $M(R)$ is dense in $\mathscr{R}$-Spec $R[M]$, thus a fortiori dense in $\mathscr{R}$-Specm $R[M]$. In addition, by (2.7) this latter space is a compact space. We therefore see that $\mathscr{R}$-Specm $R[M]$ is a kind of a geometric compactification of $M(R)$.

We next consider ultrafilters $F$ of semialgebraic subsets of $M(R)$ and their associated ordering $\alpha \in \mathscr{R}$-Spec $R[M]$. As $\mathscr{R}$-Specm $R[M]$ is compact, $F$ has a limit point in this space. In fact, it will turn out that $F$ has exactly one limit point, namely the maximal specialization of $\alpha$. But before proving this, we first have to give a new description of the correspondence between ultrafilters and elements of $\mathscr{R}$-Spec $R[M]$ since, from now on, we are dealing with orderings $\alpha$ rather than with the pairs $(\mathfrak{y}, \bar{P})$ of $\S 1$. Using only the definitions, we check the following facts: If $\alpha \in \mathscr{R}$-Spec $R[M]$ is given then it is associated to the ultrafilter $F$ which is generated by the sets $\{f=0\}$ for $f \in \mathfrak{y}_{\alpha}$ and $\{g>0\}$ for $g \in \alpha \backslash(-\alpha)$. Conversely, if $F$ is given, then $\alpha=\{f \in R[M] \mid\{f \geqslant 0\} \in F\}$ is the corresponding ordering of $R[M]$.

(3.1) Proposition. If $F$ is associated to the ordering $\alpha \in \mathscr{R}$-Spec $R[M]$ then the maximal specialization of $\alpha$ is the unique limit point of $F$ in $\mathscr{R}$-Specm $R[M]$.

Proof. Let $\beta$ be a limit point of $F$ in $\mathscr{R}$-Specm $R[M]$. In view of (2.6), we need only show $\alpha \subset \beta$. In case $\alpha \not \subset \beta$, we find $f$ with $f(\alpha) \geqslant 0$ but $f(\beta)<0$. Now the set $S=\{x \in M(R) \mid f(x) \geqslant 0\}$ belongs to $F$, and $U=\{-f>0\}$ is a neighborhood of $\beta$. Clearly $S \cap U=\varnothing$, contradicting that $\beta$ is a limit point of $F$. 
In $\$ 1$ we studied the center of an order $P$ of $F$. In general, not every order had a center. We now can introduce the center $c(P)$ of any order $P$ by defining it to be the limit of $F(P)$ in $\mathscr{R}$-Specm $R[M]$. Note that $c(P)$ is the maximal specialization of $A \cap P$ and that this definition coincides with the previous one if $P$ already has a center in $M(R)$.

By merely repeating the arguments in the proof of (1.10) we obtain the final version of Dubois' characterization of centers of orderings.

(3.2) THEOREM. The set of centers of orders in $\mathscr{R}$-Specm $R[M]$ is the closure of $M(R)_{\text {reg }}$ in $\mathscr{R}$-Specm $R[M]$.

We now return ot the embedding $\Phi: M(R) \rightarrow \mathscr{R}$-Spec $R[M]$. As already mentioned, one has the following

(3.3) THEOREM. The assignment $C \mapsto C \cap M(R)$ is a lattice isomorphism between the lattice of constructible subsets of $\mathscr{R}$-Spec $R[M]$ and the lattice of semialgebraic subsets of $M(R)$.

Proof. This map is clearly a lattice homomorphism, and it is surjective as remarked in the beginning of this section. Thus, it remains to prove that $C \cap M(R)=\varnothing$ implies $C=\varnothing$. But this statement is nothing else than the Artin-Lang homomorphism theorem, as we will see. Without loss of generality we may assume $C=\left\{f=0, g_{1}>0, \ldots, g_{r}>0\right\} \neq \varnothing$ and we want to show $C \cap M(R) \neq \varnothing$. Pick $\alpha \in C$, then we get $f \in \mathfrak{y}_{\alpha}$ and $\bar{g}_{1}, \ldots, \bar{g}_{r} \in \bar{\alpha} \backslash\{0\}$ in $k\left(\mathfrak{y}_{\alpha}\right)$. Now by (1.3), we find a homomorphism $\varphi: R[M] / \mathfrak{y}_{\alpha} \rightarrow R$ with $\varphi\left(\bar{g}_{i}\right)>0, i=1, \ldots, r$. Considering $R[M] \stackrel{\pi_{\alpha}}{\rightarrow} R[M] / \mathfrak{y}_{\alpha} \stackrel{\varphi}{\rightarrow} R$ we obtain $x \in$ $M(R) \cap C$.

By this last theorem, there is, for a given semialgebraic set $S \subset M(R)$, a unique constructible set $C \subset \mathscr{R}$-Spec $R[M]$ with $C \cap M(R)=S$. That there is at least one is a trivial remark since one may use the description of $S$ to define $C$. In view of this, the main feature of (3.3) is the independence of the chosen description of $S$; any two descriptions of $S$ will result in the same constructible set $C$.

This uniquely determined constructible subset $C$ will be denoted by $\tilde{S}$. Using (3.3) one sees that $\tilde{S}$ is the smallest constructible subset of $\mathscr{R}$-Spec $R[M]$ containing $S$. Moreover, if $\alpha$ corresponds to the ultrafilter $F$, then $\alpha \in \tilde{S}$ iff $S \in F$. This follows directly from the way $F$ is related to $\alpha$ and the above description of $\tilde{S}$ in terms of $S$.

Next, we are going to study the topological properties of the mapping $S \mapsto \tilde{S}$. Since $M(R)$ inherits the subspace topology of $\mathscr{R}-\operatorname{Spec} R[M]$, it is obvious that, given semialgebraic subsets $S \subset T, S$ is open (respectively closed) in $T$ if $\tilde{S}$ is open (resp. closed) in $\tilde{T}$. In fact we will prove more: namely, that $S$ is open in $T$ iff $\tilde{S}$ is open in $\tilde{T}$.

This is much harder than to derive (3.3). In particular, we will make use of the "base extension of semialgebraic sets". This is an important operation, in particular, in the work of Delfs and Knebusch, cf. [DK2]. Let $R \subset R^{\prime}$ be two real closed fields and let $S \subset M(R)$ be any semialgebraic set. Fix a presentation $R[M]=R\left[T_{1}, \ldots, T_{n}\right] /\left(F_{1}, \ldots, F_{m}\right)$. This means that we regard $M(R)$ as 
a subset of $R^{n}$ defined by the equations $F_{1}=0, \ldots, F_{m}=0$. This point of view will be adhered to in the sequel.

Accordingly, $S$ can then be described as follows:

$$
\begin{aligned}
S=\bigcup_{i=1}^{s}\left\{x \in R^{n} \mid F_{1}(x)=\cdots=\right. & F_{m}(x)=0, f_{i}(x)=0, \\
& \left.g_{i 1}(x)>0, \ldots, g_{i r_{i}}(x)>0\right\},
\end{aligned}
$$

where $f_{i}, g_{i 1}, \ldots, g_{i r_{1}} \in R\left[T_{1}, \ldots, T_{n}\right]$. Using such a description we define the base extension $S\left(R^{\prime}\right)$ as

$$
\begin{array}{r}
S\left(R^{\prime}\right)=\bigcup_{i=1}^{s}\left\{x \in R^{\prime n} \mid F_{1}(x)=\cdots=F_{m}(x)=0, f_{i}(x)=0,\right. \\
\left.g_{i 1}(x)>0, \ldots, g_{i r_{i}}(x)>0\right\} .
\end{array}
$$

Clearly, the question arises whether $S\left(R^{\prime}\right)$ is independent of all the choices we have made. In fact, it turns out that $S\left(R^{\prime}\right)$ is well defined. The proof of this claim is a further instance where model theory naturally applies. In particular, we make use of the model completeness of the theory of real closed fields. This asserts that for any pair of real closed fields $R^{\prime} \supset R$ a system of polynomial equalities and inequalities with coefficients in $R$ that has a solution in $R^{\prime}$ also has a solution in $R$. Hence we get

(3.4) Proposition. $S\left(R^{\prime}\right)$ is well defined relative to a fixed description of $M(R)$.

Even more is true. One can also eliminate the dependence on a fixed description of $M(R)$. However, then one has to make use of semialgebraic morphism, see e.g. [DK2]. For our purposes, the preliminary version (3.4) is general enough.

(3.5) Proposition. Let $S, T$ be semialgebraic subsets of $M(R)$. Then the following statements hold.

(i) $S \subset T$ iff $S\left(R^{\prime}\right) \subset T\left(R^{\prime}\right)$,

(ii) if $S \subset T$, then $S$ is open (respectively closed) in $T$ iff $S\left(R^{\prime}\right)$ is open (respectively closed) in $T\left(R^{\prime}\right)$.

Proof. Here again, the proof follows from the model completeness of the theory of real closed fields. Let $S$ and $T$ be given by the formulas $\sigma$ and $\tau$ in the language of ordered fields with constants in $R$. Then $S \subset T$ is expressed by $\sigma \rightarrow \tau$, and $S \subset T, S$ open in $T$, by

$$
\sigma \rightarrow \tau \wedge(\forall x)(\exists \varepsilon)(\forall y)\left[\left(\varepsilon>0 \wedge \sigma(x) \wedge \tau(y) \wedge d(x, y)^{2}<\varepsilon\right) \rightarrow \sigma(y)\right] .
$$

An analogous statement expresses $S \subset T, S$ closed. Then model completeness gives the desired conclusion.

There is a close connection between the two operations of extending the base and forming $\tilde{S}$. Let $R[M]=R\left[x_{1}, \ldots, x_{n}\right], x_{i}$ the $i$ th coordinate function. Given $\alpha \in \mathscr{R}$-Spec $R[M]$ we set $\bar{x}_{i}=\pi_{\alpha}\left(x_{i}\right) \in k(\alpha)$. With these notations we have the readily checked

(3.6) Proposition. If $S \subset M(R)$ is a semialgebraic set then $\alpha \in \tilde{S}$ iff $\left(\bar{x}_{1}, \ldots, \bar{x}_{n}\right) \in S(k(\alpha))$. 
In view of this result one may regard the real spectrum of a ring as an object which comprises and "condenses" various real closed fields related to the ring. Consequently, the theory of the real spectrum is a theory of a somewhat extended class of real closed fields, and this is the reason why, quite naturally, model theory applies.

We are now prepared to prove one of the most fundamental theorems of semialgebraic geometry. Several applications will show its central importance.

(3.7) TheOREM. Let $S, T$ be semialgebraic sets in $M(R)$ and assume $S \subset T$. Then $S$ is open (respectively closed) in $T$ if and only if $\tilde{S}$ is open (respectively closed) in $\tilde{T}$.

Proof. Since $\overline{T \backslash S}=\tilde{T} \backslash \tilde{S}$ we need only consider the case that $S$ is open in $T$. In order to prove that $\tilde{S}$ is open in $\tilde{T}$ we may appeal to (2.11) since $\tilde{S}$ and $\tilde{T}$ are clopen in the Tychonoff topology by (2.2). Hence, we have to show that $\tilde{S}$ is closed under generalizations relative to $\tilde{T}$. Thus, assume $\alpha \in \tilde{T}, \beta \in \tilde{S}$, $\alpha \subset \beta$ are given. We have to prove $\left(\pi_{\alpha}\left(x_{1}\right), \ldots, \pi_{\alpha}\left(x_{n}\right)\right)=: x_{\alpha} \in S(k(\alpha))$, in view of (3.6). By (2.9) we face the following situation where $A=R[M]$ :

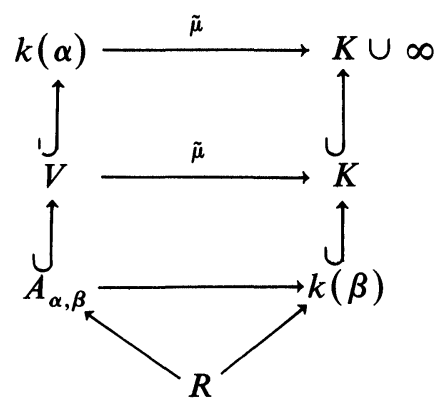

where $V$ is the convex closure of $A_{\alpha, \beta}$ in $k(\alpha)$ and $\tilde{\mu}$ extends the canonical $\operatorname{map} A_{\alpha, \beta} \rightarrow k\left(\mathfrak{y}_{\beta}\right) \rightarrow k(\beta)$.

As $k(\alpha)$ is real closed the valuation ring $V$ is henselian [PC]. Consequently, $\tilde{\mu}$ admits a section $s: K \stackrel{\rightarrow}{\rightarrow} K^{\prime} \subset V$ [PC], $s$ is the identity on $R$. By assumption and (3.6) we have $\left(\pi_{\beta}\left(x_{1}\right), \ldots, \pi_{\beta}\left(x_{n}\right):=x_{\beta} \in S(k(\beta)) \subset S(K) . S(K)\right.$ is open in $T(K)$ by (3.5)(i). We therefore find $\varepsilon \in K$ with $\varepsilon>0$ and

$$
(\forall y \in T(K))\left[\left(\tau(y) \wedge d\left(x_{\beta}, y\right)^{2}<\varepsilon\right) \rightarrow \sigma(y)\right],
$$

where we have assumed as before that $S$ and $T$ are described by $\sigma$ and $\tau$, respectively.

Denote the formula in the square brackets by $\tau\left(x_{\beta}, \varepsilon, y\right)$. Since the section $s$ is order-preserving we get that $K^{\prime}$ satisfies $\tau\left(s\left(x_{\beta}\right), s(\varepsilon), y\right)$ which, by model completeness, implies that also $k(\alpha)$ satisfies $\tau\left(s\left(x_{\beta}\right), s(\varepsilon), y\right)$. Now assume $x_{\alpha} \notin S(k(\alpha))$. Then necessarily

$$
d\left(s\left(x_{\beta}\right), x_{\alpha}\right)^{2} \geqslant s(\varepsilon) .
$$


Applying the order-preserving place $\tilde{\mu}$ to this inequality we see

$$
d\left(\tilde{\mu} s\left(x_{\beta}\right), \tilde{\mu}\left(x_{\alpha}\right)\right)^{2} \geqslant \tilde{\mu} s(\varepsilon),
$$

since all entries are elements of $V$. But $\tilde{\mu} s=\mathrm{id}$ and $\tilde{\mu}\left(x_{\alpha}\right)=x_{\beta}$, which gives the contradiction.

This beautiful proof is due to L. v. d. Dries. In [Dr] he presented the model-theoretic background of this approach.

As a first application of (3.7) we will derive the so-called finiteness theorem [De, Bru1, C] of semialgebraic geometry. This theorem deals with the description of open semialgebraic sets. Assume $S, T$ are semialgebraic sets and that $S$ is open in $T$. By (3.7) we see that $\tilde{S}$ is open in $\tilde{T}$. Now (2.12) applies and states that $\tilde{S}$ is open-constructible in $\tilde{T}$. This means that $\tilde{S}$ is a finite union of sets of the type $\left\{\alpha \in \mathscr{R}\right.$-Spec $\left.R[M] \mid \alpha \in \tilde{T}, f_{1}(\alpha)>0, \ldots, f_{r}(\alpha)>0\right\}$. The latter set equals $\tilde{U}$, where $U=\left\{x \in M(R) \mid x \in T, f_{1}(x)>0, \ldots, f_{r}(x)>0\right\}$. Thus $\tilde{S}=\tilde{U}_{1} \cup \cdots \cup \tilde{U}_{s}$ where $U_{i}$ is of the type just described. Going back to $M(R)$, we obtain $S=U_{1} \cup \cdots \cup U_{r}$, i.e. $S$ can be described by a finite disjunction of "open conditions" $f_{1}>0, \ldots, f_{r}>0$ relative to $T$. This is the

(3.8) (FINITENESS THEOREM). If $S$ and $T$ are semialgebraic subsets of $M(R)$ and if $S$ is open in $T$ then $S$ is a finite union of open subsets of the type

$$
\left\{x \in T \mid f_{1}(x)>0, \ldots, f_{r}(x)>0\right\},
$$

where $r \in \mathbf{N}, f_{1}, \ldots, f_{r} \in R[M]$.

One should notice that, using (3.8), one can derive an analogous statement for the case that $S$ is closed in $T$. It says that a closed $S$ can be described by a finite disjunction of "closed conditions" $f_{1} \geqslant 0, \ldots, f_{r} \geqslant 0$.

In the proof of the finiteness theorem we applied the properties of the real spectrum to obtain results on semialgebraic sets. We next reverse the order of arguments to study the connected components of a constructible set in $\mathscr{R}$-Spec $R[M]$. We will start with the decomposition of a semialgebraic set into semialgebraic components [CC1, 5.5; DK1, §10ff; Bru1, p. 260 ff]. A semialgebraic set $S \subset M(R)$ is called semialgebraically connected if it has no decomposition $S=S_{1} \dot{\cup} S_{2}$ into disjoint open semialgebraic subsets $S_{1}, S_{2}$. As a consequence of (3.8) we obtain

(3.9) Proposition. $S$ is semialgebraically connected if and only if $\tilde{S}$ is a connected topological space.

Proof. If $S$ is semialgebraically connected but $\tilde{S}$ is not (as a topological space) we have $\tilde{S}=U_{1} \dot{\cup} U_{2}, U_{i} \neq \varnothing, U_{i}$ open in $\tilde{S}$. In the Tychonoff topology, $\tilde{S}$ is clopen, hence $U_{i}$ is also clopen. Again by (2.2), each $U_{i}$ is constructible, thus $U_{i}=\tilde{S}_{i}$ for some semialgebraic set $S_{i} \subset S, S_{i} \neq \varnothing$. We get $S=S_{1} \cup S_{2}$ with $S_{i}$ open in $S$ by (3.7), a contradiction. The converse follows from (3.7).

The main importance of (3.8) lies in the fact that the real spectrum, with its topology, remedies certain defects arising from the "poor" topology of an arbitrary real closed field $R$. Namely, if $R \neq \mathbf{R}$, then $R$ and, consequently, 
every semialgebraic set is totally disconnected. Thus it is useless to define connected components by referring to the strong topology. However, one may introduce, as we did, the notion of a semialgebraically connected semialgebraic set. It is then a remarkable fact that this notion corresponds to the usual notion of a connected topological space if we pass to the real spectrum.

Even more is true. It is known that every semialgebraic set $S$ decomposes into a finite number of open, semialgebraically, connected, semialgebraic subsets, its so-called semialgebraic connected components $S_{1}, \ldots, S_{r}$, see [Bru1, p. $260 \mathrm{ff}$; CC1, 5.5; DK1, $\$ 10 \mathrm{ff}]$. By (3.7) and (3.9), each $\tilde{S}_{i}$ is an open connected subspace. Because of $\tilde{S}=\tilde{S}_{1} \dot{\cup} \ldots \dot{\cup} \tilde{S}_{r}$ we see that the $\tilde{S}_{i}$, $i=$ $1, \ldots, r$, are the connected components of $\tilde{S}$. Taking into account that every constructible subset is of the type $\tilde{S}, S$ semialgebraic, we obtain the following

(3.10) THEOREM. Every constructible subset $C$ of $\mathscr{R}$-Spec $R[M]$ has a finite number of connected components and, if $C=\tilde{S}, S$ semialgebraic, the connected components of $C$ correspond bijectively to the semialgebraic connected components of $S$.

REMARKS. (i) It would be really nice to have a proof of (3.10) which does not start on the semialgebraic side and then passes to the real spectrum. Such a proof should work completely inside $\mathscr{R}$-Spec $R[M]$ and then transfer the results to $M(R)$ in order to derive the finiteness of $\pi_{0}(S)$. An example of this type of proof is given by the proof of (3.8).

(ii) Theorem (3.7) is also very important if one studies sheaf theory on semialgebraic sets. By (3.7), one can relate sheaves on semialgebraic sets to sheaves on constructible sets. This change in the point of view has some advantages. Details can be found in H. Delfs's paper [D].

We want to conclude this section by briefly describing some of the nice and important results of Bröcker on the number of equalities and inequalities needed to describe a semialgebraic set, cf. [Br3, Rr4]. For the sake of brevity we will confine ourselves to the question of describing an open semialgebraic set

$$
S\left(f_{1}, \ldots, f_{r}\right)=\left\{x \in M(R) \mid f_{1}(x)>0, \ldots, f_{r}(x)>0\right\} .
$$

The problem is then the following: Is there a bound $s(M)$ depending only on $M$ such that every open semialgebraic set $S\left(f_{1}, \ldots, f_{r}\right), f_{1}, \ldots, f_{r} \in R[M]$, $r \in \mathrm{N}$,can already be described by $s=s(M)$ inequalities, i.e.

$$
S\left(f_{1}, \ldots, f_{r}\right)=S\left(g_{1}, \ldots, g_{s}\right), \quad g_{1}, \ldots, g_{s} \in R[M] .
$$

\section{Bröcker proves}

(3.11) THEOREM $[\mathrm{Br} 3,6.4]$. There is a bound $s(M)$ and in fact

$$
s(M) \leqslant\left\{\begin{array} { l } 
{ 1 \cdot 3 \cdot 5 \cdots n } \\
{ 2 \cdot 4 \cdot 6 \cdots n }
\end{array} \quad \text { if } n = \operatorname { d i m } M \text { is } \left\{\begin{array}{l}
\text { odd }, \\
\text { even. }
\end{array}\right.\right.
$$

It is not possible to present his ingenious proof here. However, we shall sketch the main line of the proof: Recall that after (1.12) we derived the diagram

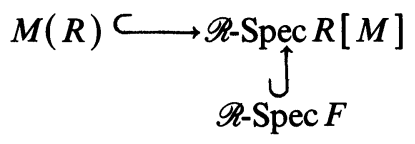


where $F=R(M)$ is the function field of $M$. Now consider $S=S\left(f_{1}, \ldots, f_{r}\right)$, as above. Then pass to $\tilde{S} \subset \mathscr{R}$-Spec $R[M]$. By (3.7) we know that $\tilde{S}$ is open in $\mathscr{R}$-Spec $R[M]$. Next study $\tilde{S} \cap \mathscr{R}$-Spec $F=: U$. We have

$$
U=\left\{P \in \mathscr{R} \text {-Spec } F \mid f_{1}, \ldots, f_{r} \in P\right\} .
$$

The latter set belongs to the so-called Harrison basis of $\mathscr{R}$-Spec $F$ and is often denoted by $H\left(f_{1}, \ldots, f_{r}\right)$. If we had $S=D\left(g_{1}, \ldots, g_{s}\right)$ then also $H\left(f_{1}, \ldots, f_{r}\right)$ $=H\left(g_{1}, \ldots, g_{s}\right)$. Consequently, as a first step to a solution of our problem, we would have to solve the corresponding question for the description of the open sets $H\left(f_{1}, \ldots, f_{r}\right)$ in $\mathscr{R}$-Spec $F$. This is a problem that belongs to the theory of formally real fields and has been solved in the 70 s by the theory of quadratic forms over formally real fields. Using this, we have solved our problem on the level of $\mathscr{R}$-Spec $F$. However, we have to resolve the problem in $M(R)$ instead of $\mathscr{R}$-Spec $F$. This means that one has to pull back the information from $\mathscr{R}$-Spec $F$ to $M(R)$ via $\mathscr{R}-\operatorname{Spec} R[M]$. This is not an easy task. The main difficulty is that there are many open sets in $\mathscr{R}$-Spec $R[M]$ having the same intersection with $\mathscr{R}$-Spec $F$. Any two of them differ only by an algebraic subset of lower dimension, thus some sort of induction will apply. But, in carrying out the details, a great deal of subtle arguments have to be made. All in all, Bröcker's proof is not easy.

Nevertheless, this proof demonstrates, once again, that general results from the theory of ordered fields can be successfully applied to real algebraic geometry via the notion of the real spectrum.

4. On abstract semialgebraic geometry. In this section we are going to touch upon a possible foundation of abstract semialgebraic geometry. This means that we want to build up the theory using only constructible subsets of the real spectra of some rings. We thus work "abstractly" rather than dealing with the "concrete" semialgebraic subsets of some $M(R)$. This procedure, of course, parallels the one in classical algebraic geometry where one bases the theory on the notion of an affine scheme, $\operatorname{Spec} A$. To develop such an abstract theory requires, as is well known, a lot of work and space. Consequently we will only present the very beginnings. For more details, we refer to [R, Bru2, S2, S3, D]. The paper [S2] of N. Schwartz is especially highly recommended. In particular, we will present here his definition of the structure sheaf on a constructible set.

Before turning to this topic, however, we want to derive various Null- and Positivstellensätze in the abstract setting of the real spectrum of a ring. This is done to emphasize the fact that it is the real spectrum where they can be proved most naturally and easily. Again, by applying the correspondence between semialgebraic and constructible subsets, we will then obtain the Nulland Positivstellensatz for semialgebraic sets. See also [St, Br1, CT, L2].

Let $A$ denote, as in $\S 2$, an arbitrary ring. We want to study the existence of orderings $\alpha$ of $A$ with certain prescribed properties. As will be seen from later applications, the prescribed properties always amount to assuming that a certain quadratic semiring $T$ is contained in $\alpha: T \subset \alpha$. Here, a quadratic semiring is any subset of $A$ containing $A^{2}$ and closed under addition and multiplication. Thus $\sum A^{2}=\left\{\sum_{1}^{n} x_{i}^{2} \mid n \in \mathbf{N}, x_{1}, \ldots, x_{n} \in A\right\}$ is the smallest quadratic semiring in $A$. 
(4.1) Proposition. Let $T$ be a quadratic semiring. There is an ordering $\alpha \in \mathscr{R}$-Spec $A$ with $T \subset \alpha$ iff $-1 \notin T$.

We have as an obvious corollary

(4.2) THEOREM. $\mathscr{R}$-Spec $A \neq \varnothing$ iff $-1 \notin \sum A^{2}$.

Proof of (4.1). Clearly, $-1 \notin T$ if $T \subset \alpha$. Conversely, assume $-1 \notin T$. Set $H=1+T . H$ is a multiplicative semigroup, and we have $H \cap\{0\}=\varnothing$. By Krull's theorem we find a prime ideal $\mathfrak{y}$ which is maximal among all ideals $a$ with $H \cap \mathfrak{a}=\varnothing$. Let $K=\operatorname{Quot}(A / \mathfrak{y})$ and $\bar{H}=\{\bar{s}=s+\mathfrak{y} \mid s \in H\}$. We claim that $K$ admits an order $\bar{P}$ with $\bar{H} \subset \bar{P}$. To prove this we have to show that the quadratic semiring of $K$ generated by $\bar{H}$ does not contain -1 , see [B, (1.2) ff]. Assume on the contrary $-1=\sum_{1}^{n} \bar{s}_{i} z_{i}^{2}, z_{i} \in K^{\times}, s_{i} \in H$; then there are $y, x_{1}, \ldots, x_{n} \in A$ with $y \notin \mathfrak{y}$ and $y^{2}+\sum_{1}^{n} s_{i} x_{i}^{2} \in \mathfrak{y}$. Now, because of the maximality of $\mathfrak{y}$ and $y \notin \mathfrak{y}$, there are $a \in \mathfrak{y}, b \in A, s \in H$ with $1+s=a+$ by. Then $b^{2} y^{2} \equiv(1+s)^{2} \bmod \mathfrak{y}$, and from $b^{2} y^{2}+b^{2} \sum s_{i} x_{i}^{2} \in \mathfrak{y}$ we get $t=(1+s)^{2}+\sum s_{i}\left(b x_{i}\right)^{2} \in \mathfrak{y}$. Clearly, $t \in 1+T=H$, which is a contradiction. Having now this order $\bar{P} \supset \bar{H}$ we set $\alpha=\{a \in A \mid a+\mathfrak{y} \in \bar{P}\}$. Then $\alpha \in \mathscr{R}$-Spec $A$ and $H \subset \alpha$.

We next turn to the abstract versions of certain Null- and Positivstellensätze. To have a concrete example consider a semialgebraic set $S$ given by

$$
\begin{array}{r}
S=\left\{x \in M(R) \mid f_{1}(x)=\ldots=f_{r}(x)=0, g_{1}(x) \geqslant 0, \ldots, g_{s}(x) \geqslant 0,\right. \\
\left.h_{1}(x)>0, \ldots, h_{t}(x)>0\right\} .
\end{array}
$$

It is natural to ask for a description of functions $f \in R[M]$ with $f=0$ or $f \geqslant 0$ or $f>0$ on $S$, in terms of the given data $f_{1}, \ldots, f_{r}, g_{1}, \ldots, g_{s}, h_{1}, \ldots, h_{t}$. Passing to the abstract setting, we start off with a quadratic semiring $T$ and ask for a description of $f \in A$ such that $f(\alpha)=0$ (or $f(\alpha) \geqslant 0$ or $f(\alpha)>0$ ) whenever $T \subset \alpha$. In the next three statements (4.3)-(4.5) the ring $A$ and quadratic semiring $T$ are kept fixed.

(4.3) NullSTELLENSATZ. The following statements are equivalent:

(i) $f(\alpha)=0$ for all $\alpha$ with $T \subset \alpha$,

(ii) $f^{2 k}+t=0$ for some $k \in \mathbf{N}, t \in T$.

Proof. (i) $\Rightarrow$ (ii) We consider the quadratic semiring

$$
T_{f}:=\left\{\frac{t}{f^{2 l}} \mid l \in \mathbf{N}, t \in T\right\}
$$

in $A_{f}$. If $\alpha \in \mathscr{R}$-Spec $A_{f}$ is given we write, by abuse of notation,

$$
\alpha \cap A=\left\{a \in A \mid \frac{a}{1} \in \alpha\right\} \in \mathscr{R} \text {-Spec } A \text {. }
$$

If $-1 \notin T_{f}$ then, by 4.1 , there is an $\alpha$ with $T_{f} \subset \alpha$. But then $f(\alpha \cap A) \neq 0$. Hence $-1 \in T_{f}$, which leads to

$$
f^{n}\left(1+\frac{t}{f^{2 l}}\right)=0
$$

for some $n, l \in \mathbf{N}, t \in T$. We may choose $n$ even to derive (ii).

(ii) $\Rightarrow$ (i) Since $t(\alpha) \geqslant 0$ and $(f(\alpha))^{2 k} \geqslant 0$ we get $f(\alpha)=0$. 
(4.4) STRict Positivstellensatz. The following statements are equivalent:

(i) $f(\alpha)>0$ for all $\alpha$ with $T \subset \alpha$,

(ii) $f t=1+s$ for some $t, s \in T$.

Proof. (ii) $\Rightarrow$ (i) If $T \subset \alpha$ then $f(\alpha) t(\alpha)=1+s(\alpha), t(\alpha), s(\alpha) \geqslant 0$, hence $f(\alpha) t(\alpha)>0$ and $f(\alpha)>0$.

(i) $\Rightarrow$ (ii) Consider the quadratic semiring $T+(-f) T=T^{\prime}$. In case $-1 \notin T^{\prime}$ we would find $\alpha \in \mathscr{R}$-Spec $A$ with $T \subset \alpha,(-f)(\alpha) \geqslant 0$, i.e. $T \subset \alpha, f(\alpha) \leqslant 0$ : a contradiction. Therefore, $-1 \in T^{\prime}$, which means $f t=1+s$ for some $t, s \in T$.

(4.5) Positivstellensatz. The following statements are equivalent:

(i) $f(\alpha) \geqslant 0$ for all $\alpha$ with $T \subset \alpha$,

(ii) $f t=f^{2 k}+s$ for some $k \in \mathbf{N}, t, s \in T$.

Proof. To prove (ii) $\Rightarrow$ (i) one argues as above.

(i) $\Rightarrow$ (ii) Consider the quadratic semiring of $A_{f}$ introduced above. Then

$$
\frac{f}{1}(\alpha)>0 \text { for all } \alpha \in \mathscr{R}-\operatorname{Spec} A_{f}, T_{f} \subset \alpha \text {. }
$$

By (4.4), we get

$$
\frac{f}{1} \cdot t=1+s \text { for some } t, s \in T_{f} .
$$

Pulling this back to $A$, one obtains (ii) with possibly other $t, s$.

We are now going to derive the "concrete" version of the last results (4.3)-(4.5). This means that we will be dealing with a semialgebraic subset $S$ of some affine variety $M(R)$, where we assume that $S$ is given as described before (4.3). Then in terms of the defining functions $f_{1}, \ldots, f_{r}, g_{1}, \ldots, g_{s}, h_{1}, \ldots, h_{t}$, we want to describe all functions $f \in R[M]$ which are equal to zero, or which are strictly positive, or which are nonnegative on $S$. Furthermore, we want to give a proof which is based on our abstract solution of such problems, i.e. (4.3)-(4.5). That both questions are related is due to the correspondence between semialgebraic subsets of $M(R)$ and constructible subsets of $\mathscr{R}$-Spec $R[M]$, cf. (3.3). Thus, let $S_{1}$ denote any one of the following sets:

$$
\begin{gathered}
\{x \in M(R) \mid f(x)=0\}, \quad\{x \in M(R) \mid f(x)>0\}, \\
\{x \in M(R) \mid f(x) \geqslant 0\} .
\end{gathered}
$$

By assumption, $S \subset S_{1}$ in each of the cases we are dealing with. Since $S^{\prime} \subset S^{\prime \prime}$ implies $\tilde{S}^{\prime} \subset \tilde{S}^{\prime \prime}$ if $S^{\prime}$ and $S^{\prime \prime}$ are semialgebraic, we conclude $\tilde{S} \subset \tilde{S}_{1}$, i.e. $f=0$ on $\tilde{S}$ or $f>0$ on $\tilde{S}$ or $f \geqslant 0$ on $\tilde{S}$ respectively. So, in order to apply (4.3) through (4.5) we have to describe $\tilde{S}$ by a certain quadratic semiring. To this end, still using the previous description of $S$, let a be the ideal of $R[M]$ generated by $f_{1}, \ldots, f_{r}, H$ be the multiplicative semigroup with unit in $R[M]$ generated by $h_{1}, \ldots, h_{t}$ and $T$ be the quadratic semiring in $R[M]$ generated by $R[M]^{2}$ and the functions $g_{1}, \ldots, g_{s}, h_{1}, \ldots, h_{t}$. Next set $B=R[M] / \mathfrak{a}$, $\bar{r}=r+\mathfrak{a}$ for $r \in R[M], \bar{H}=\{\bar{h} \mid h \in H\}, \bar{T}=\{\bar{t} \mid t \in T\}, C=B_{\bar{H}}$, the ring of fractions with respect to $\bar{H}$, and

$$
\bar{T}_{1}:=\left\{\frac{\bar{t}}{\bar{h}} \mid \bar{t} \in \bar{T}, \bar{h} \in \bar{H}\right\} .
$$


Using the natural homomorphism

$$
q: R[M] \rightarrow C, \quad a \mapsto \frac{\bar{a}}{1}
$$

and the relation $\bar{q}\left(a\left(q_{*}(\alpha)\right)\right)=q(a)(\alpha)$ for $a \in R[M]$, cf. (2.13), we obtain

$$
\tilde{S}=q_{*}\left\{\alpha \in \mathscr{R} \text {-Spec } C \mid \bar{T}_{1} \subset \alpha\right\} \text {. }
$$

Again by using (2.13), we conclude, in our situation, that $\tilde{f}:=q(f)$ satisfies

$$
\tilde{f}(\alpha)=0 \quad(\text { or } \tilde{f}(\alpha)>0 \text { or } \tilde{f}(\alpha) \geqslant 0 \text { respectively) }
$$

for $\alpha \in \mathscr{R}$-Spec $C$ with $\bar{T}_{1} \subset \alpha$. Now, the previous results (4.3)-(4.5) can be applied and we get

(4.7) NULLSTELLENSATZ. The following statements are equivalent:

(i) $f(x)=0$ for all $x \in S$,

(ii) $h f^{2 k}+t \in$ a for some $h \in H, k \in \mathbf{N}, t \in T$.

Proof. (ii) $\Rightarrow$ (i) First note that, if $x \in S, a(x)=0$ for $a \in \mathfrak{a}, b(x) \geqslant 0$ for $b \in T, c(x)>0$ for $c \in H$. This proves (ii) $\Rightarrow$ (i). To prove the converse, (i) $\Rightarrow$ (ii), we start off with the fact just derived that

$$
\frac{\bar{f}}{1}(\alpha)=0 \text { for all } \alpha \in \mathscr{R} \text {-Spec } C \text { with } \bar{T}_{1} \subset \alpha .
$$

From (4.3) we get

$$
\left(\frac{\bar{f}}{1}\right)^{2 k}+\frac{\bar{t}}{\bar{h}}=0 \quad \text { for certain } k \in \mathbf{N}, h \in H, t \in T .
$$

Pulling this back to $R[M]$ we get the desired conclusion (with possibly other $h$ and $t$ ).

The way of reasoning just presented also applies to the other situations: $f>0$ on $S$ and $f \geqslant 0$ on $S$. We get

(4.8) Strict Positivstellensatz. The following statements are equivalent:

(i) $f(x)>0$ for all $x \in S$,

(ii) $f t \equiv h+s \bmod$ a for some $t, s \in T, h \in H$.

(4.9) Positivstellensatz. The following statements are equivalent:

(i) $f(x) \geqslant 0$ for all $x \in S$,

(ii) $f t \equiv h f^{2 k}+s \bmod$ a for some $k \in \mathbf{N}, t, s \in T, h \in H$.

We come next to the main part of this section, namely N. Schwartz's construction of a structure sheaf on a constructible set $[\mathbf{S 2}, \mathbf{D}]$. Prior to his definition, a structure sheaf on the real spectrum was already introduced by M.-F. Roy, a detailed account of which is presented in [R]. Here, however, we will concentrate on the sheaf of "abstract semialgebraic functions" defined by Schwartz. As its name already indicates, with this sheaf the concept of semialgebraic function is transferred to an abstract setting. First recall the definition of a semialgebraic map $f: S_{1} \rightarrow S_{2}$, where $S_{i}$ is a semialgebraic subset of an affine variety $M_{i}(R), i=1,2$. According to [DK2, Definition 3, 
p. 179] a map $f: S_{1} \rightarrow S_{2}$ is called semialgebraic if $f$ is continuous (with respect to the strong topology) and the graph of $f$ is a semialgebraic subset of $S_{1} \times S_{2}$.

In dealing with a constructible subset $X$ of $\mathscr{R}$-Spec $A$, we clearly want that at least the elements of $A$ are to be regarded as abstract semialgebraic functions. Given $f \in A$, we may consider, as we did before, $f$ as a "function" on $X$ with values in the real closed fields $k(\alpha), \alpha \in X$, introduced before (2.8). More precisely, we assign to $f$ the element

$$
\tilde{f}=(f(\alpha))_{\alpha \in X} \in \prod_{\alpha \in X} k(\alpha)=: \Pi .
$$

The assignment $f \mapsto \tilde{f}$ is a ring homomorphism $A \rightarrow \Pi$ with image denoted by $\tilde{A}$. Taking this point of view, we next look for overrings of $\tilde{A}$ in $\Pi_{\alpha \in X} k(\alpha)$; one of these rings will later on be declared the ring of abstract semialgebraic functions on $X$. In order to impose natural conditions on subrings of $\Pi$ we first interpret its elements as certain sections of $p=j_{*}: \mathscr{R}$-Spec $A[T] \rightarrow$ $\mathscr{R}$-Spec $A$ where $A[T]$ is the polynomial ring in the variable $T$ and $j: A \rightarrow A[T]$ is the inclusion. To this end we recall the homeomorphism

$$
\varphi_{*}^{-1}(\alpha) \stackrel{i_{*}}{\leftarrow} \mathscr{R}-\operatorname{Spec}\left(k(\alpha) \otimes_{A} B\right)
$$

of Theorem (2.17). In the present situation we have

$$
p^{-1}(\alpha) \stackrel{i_{*}}{\leftarrow} \mathscr{R}-\operatorname{Spec} k(\alpha)[T] .
$$

Now, given $a=(a(\alpha))_{\alpha \in X} \in \Pi$, we obtain, for any $\alpha \in X$, the element $\left(\mathfrak{m}_{a(\alpha)}, k(\alpha)^{2}\right)=: \beta_{\alpha}$ in $\mathscr{R}-\operatorname{Spec} k(\alpha)[T]$. Then $s_{a}(\alpha):=i_{*}\left(\beta_{\alpha}\right) \in p^{-1}(\alpha)$. Thus we have defined a section $s_{a}: X \rightarrow p^{-1}(X) \subset \mathscr{R}$-Spec $A[T]$ of $p: p^{-1}(X) \rightarrow X$. The question arises how to characterize these sections among all sections of $p$. Here we have

(4.10) Proposition. The assignment $a \mapsto$ is a bijection between $\prod_{\alpha \in X} k(\alpha)$ and the set of all sections $s: X \rightarrow p^{-1}(X)$ of $p$ such that, for all $\alpha \in X$, the point $s(\alpha)$ is constructible in $p^{-1}(\alpha)$.

REMARK. By abuse of notation, a point $\beta$ is called constructible if $\{\beta\}$ is constructible, and $p^{-1}(\alpha)$ is written instead of $p^{-1}(\{\alpha\})$.

Proof. We first note that the only constructible points of $\mathscr{R}$-Spec $k[T], k$ a real closed field, are the points of $k$, i.e. the orderings $\left(\mathrm{m}_{x}, k^{2}\right), x \in k$. This can be seen directly or by using the correspondence between constructible and semialgebraic sets, see Theorem (3.3). Next we take into account, as mentioned in the proof of $(2.17)$, that $i_{*}: \varphi_{*}^{-1}(\alpha) \leftarrow \mathscr{R}-\operatorname{Spec}\left(k(\alpha) \otimes_{A} B\right)$ constitutes a homeomorphism with respect to the Tychonoff topology and that a subset is constructible iff it is clopen in the Tychonoff topology. Putting all this together we obtain the desired result.

In the sequel we will often define orderings of $A\left[T_{1}, \ldots, T_{n}\right]$ in the following way. We start off with an ordering $\alpha$ of $A$. This ordering induces the homomorphism $\pi_{\alpha}: A \rightarrow k(\alpha)$. The extensions of $\pi_{\alpha}$ to $A\left[T_{1}, \ldots, T_{n}\right]$ with values in some real closed field $L \supset k(\alpha)$ are in one-to-one correspondence 
with prescribed values $l_{1}, \ldots, l_{n}$ for $T_{1}, \ldots, T_{n}$ in $L$. Thus, given $L$ and $l_{1}, \ldots, l_{n} \in L$ we obtain $\hat{\pi}_{\alpha}$ by requiring $\hat{\pi}_{\alpha \mid A}=\pi_{\alpha}, \hat{\pi}_{\alpha}\left(T_{i}\right)=l_{i}$. Then $\beta:=\left(\hat{\pi}_{\alpha}\right)^{*}\left(L^{2}\right)$ is an ordering of $A\left[T_{1}, \ldots, T_{n}\right]$ which extends $\alpha$. If $\alpha \in X$ and $f \in A$ are given we want to describe the section $s_{\tilde{f}}$ and the ordering $\beta=s_{\tilde{f}}(\alpha)$. We get that $\pi_{\beta}: A[T] \rightarrow k(\alpha)=k(\beta)$ is induced by $\pi_{\alpha}: A \rightarrow k(\alpha), T \mapsto f(\alpha)$. In particular, let $A=R[M]$ be the coordinate ring of an affine variety $M(R)$, $R$ a real closed field. Let $x \in M(R)$ and $\alpha:=\{g \in A \mid f(x) \geqslant 0\}(\alpha=\Phi(x)$ according to $\S 1) . A[T]$ is then the coordinate ring of $M(R) \times R$ and $\beta=s_{\tilde{f}}(\alpha)$ corresponds to the point $(x, f(x)) \in M(R) \times R$. Thus the section $s_{\tilde{f}}$, when restricted to $M(R) \subset \mathscr{R}$-Spec $R[M]$, just describes the graph of $f$.

Back in our quite general situation, we may also regard $s_{a}(X)$ as the graph of the "abstract function" $a \in \Pi k(\alpha)$. Using this interpretation we will single out the ring $C(X)$ of abstract semialgebraic functions by the following restrictions: $a \in C(X)$ iff

(i) $s_{a}: X \rightarrow p^{-1}(X)$ is continuous,

(ii) $s_{a}(X)$ is constructible and closed in $p^{-1}(X)$.

These conditions seem to be rather reasonable. However, it is by no means obvious that $C(X)$ is a subring of $\Pi_{\alpha \in X} k(\alpha)$. In fact the proof will make use of all the more difficult results obtained in this paper.

A similar definition for abstract semialgebraic functions has been given earlier by Brumfiel in [Bru2]. But he did not require $s_{a}(X)$ to be closed in $p^{-1}(X)$. It was $\mathrm{N}$. Schwartz who discovered the importance of this condition and proved the following fundamental theorem.

(4.11) THEOREM. $C(X)$ is a subring of $\Pi_{\alpha \in X} k(\alpha)$ which contains $\tilde{A}$.

The rest of this section, apart from some remarks, will be devoted to the proof of (4.11). For clarity, we are going to introduce some intermediate concepts and results.

First, call a section $s: X \rightarrow p^{-1}(X)$ "pointwise-constructible", if $s(\alpha)$ is constructible in $p^{-1}(\alpha)$ for any $\alpha \in X$. By (4.10), these sections are in one-to-one correspondence with the elements of $\prod_{\alpha \in X} k(\alpha)$; the bijection being given by $s \mapsto(T(s(\alpha)))_{\alpha \in X}$

We may therefore introduce the sum and product of pointwise-constructible sections $s$ and $t$ by setting

$$
\begin{array}{ll}
p((s+t)(\alpha))=\alpha, & T((s+t)(\alpha))=T(s(\alpha))+T(t(\alpha)), \\
p(s t(\alpha))=\alpha, & T(s t(\alpha))=T(s(\alpha)) \cdot T(t(\alpha)) .
\end{array}
$$

Secondly, we call a section $s$ "constructible" if $s(X)$ is constructible in $p^{-1}(X)$. In this case, $s(X)$ is also constructible since $p^{-1}(X)$ is constructible. Any constructible section is pointwise-constructible. This follows from $\{s(x)\}$ $=p^{-1}(x) \cap s(X)$. We therefore may consider the sum and product of constructible sections to obtain sections that are at least pointwise-constructible. However, we have:

(4.13) Proposition. With the operations (4.12) the set of constructible sections is a ring. 
Proof. We consider the $A$-algebra homomorphism

$$
\begin{aligned}
a: A[T] \rightarrow A\left[T_{1}, T_{2}\right], & T & \mapsto T_{1}+T_{2}, \\
m: A[T] \rightarrow A\left[T_{1}, T_{2}\right], & T & \mapsto T_{1} T_{2} .
\end{aligned}
$$

Starting off with two constructible sections $s_{1}, s_{2}$ we will construct a section $s=\left(s_{1}, s_{2}\right): X \rightarrow \mathscr{R}-\operatorname{Spec} A\left[T_{1}, T_{2}\right]$ with constructible image such that $a_{*} \circ s=$ $s_{1}+s_{2}$ and $m_{*} \circ s=s_{1} s_{2}$ holds. Now, in both cases the homomorphisms $a$ and $m$ turn $A\left[T_{1}, T_{2}\right]$ into a finitely presented $A[T]$-algebra, since in the case of the sum $a$, we have $A\left[T_{1}, T_{2}\right]=A\left[T_{1}+T_{2}, T_{2}\right]$, and in the case of the product one can show

$$
\left(A\left[T_{1} T_{2}\right]\right)\left[X_{1}, X_{2}\right] /\left(X_{1} X_{2}-T_{1} T_{2}\right) \stackrel{\sim}{\rightarrow} A\left[T_{1}, T_{2}\right] .
$$

Therefore, Theorem (2.1) applies and shows that $\left(a_{*}^{\circ} s\right)(X)=a_{*}(s(X))$ and $\left(m_{*} \circ s\right)(X)$ are also constructible-which is what we wanted to prove. It remains, therefore, to construct $s$. We define $s(\alpha), \alpha \in X$, by the following conditions:

$$
s(\alpha) \cap A=\alpha, \quad T_{i}(s(\alpha))=T\left(s_{i}(\alpha)\right) \text { for } i=1,2 .
$$

Denoting the $A$-algebra homomorphism $A[T] \rightarrow A\left[T_{1}, T_{2}\right], T \mapsto T_{i}$ by $\varphi_{i}$, we see that $\left(\varphi_{i}\right)_{*} s(\alpha)=s_{i}(\alpha), i=1,2$. Conversely, assume $\left(\varphi_{i}\right)_{*}(\gamma) \in s_{i}(X)$ for $i=1,2, \gamma \in \mathscr{R}-\operatorname{Spec} A\left[T_{1}, T_{2}\right]$, then $\left(\varphi_{i}\right)_{*}(\gamma)=s_{i}\left(\alpha_{i}\right)$. Since $s_{i}$ is a section, we necessarily get $\alpha_{i}=\gamma \cap A=: \alpha$, independent of $i$. This implies $T_{i}(\gamma)=$ $T\left(s_{i}(\alpha)\right)=T_{i}(s(\alpha))$, hence $\gamma \in s(X)$. Thus, we have proved $s(X)=$ $\left(\varphi_{1}\right)_{*}^{-1}\left(s_{1}(X)\right) \cap\left(\varphi_{2}\right)_{*}^{-1}\left(s_{2}(X)\right)$ and $s(X)$ is also constructible in view of Propositions (2.2) and (2.14). Finally, we have to show $a_{*} \circ s=s_{1}+s_{2}$, $m_{*} \circ s=s_{1} s_{2}$. Clearly, $p a_{*}=p m_{*}=j_{*}$ where $j: A \rightarrow A\left[T_{1}, T_{2}\right]$ is the natural inclusion. Moreover,

$$
T\left(a_{*}(s(\alpha))\right)=a(T)(s(\alpha))=\left(T_{1}+T_{2}\right)(s(\alpha))=T\left(s_{1}(\alpha)\right)+T\left(s_{2}(\alpha)\right)
$$

and

$$
T\left(m_{*}(s(\alpha))\right)=m(T)(s(\alpha))=T\left(s_{1}(\alpha)\right) \cdot T\left(s_{2}(\alpha)\right),
$$

which completes the proof.

We next have to deal with constructible sections $s$ which are continuous and have a closed image $s(X)$ in $p^{-1}(X)$. In order to show that differences and products of such sections are again of this type (i.e. that $C(X)$ is a ring), we will reformulate the latter two conditions with the help of the places $\mu_{\alpha, \beta}$ : $k(\alpha) \rightarrow K \cup \infty$, introduced in the first two sections, whenever $\alpha, \beta$ with $\alpha \subset \beta$ are given.

To avoid notational confusion in the sequel, we fix for each $\alpha \in \mathscr{R}$-Spec $A$ a real closure $k(\alpha)$ of $k\left(\mathfrak{y}_{\alpha}\right)$. Given $\alpha \subset \beta, \alpha, \beta \in \mathscr{R}$-Spec $A$, we denote by $V_{\alpha, \beta}$ the convex closure of $A_{\alpha, \beta}$ in $k(\alpha)$. More generally, we now consider all convex valuation rings $V$ of $k(\alpha)$ which dominate $A_{\alpha, \beta}$, i.e. which satisfy the following conditions:

$$
A_{\alpha, \beta} \subset V, \mathfrak{y}_{\alpha, \beta} \subset \mathfrak{m}_{V} \quad(=\text { the maximal ideal of } V) .
$$


Since convex valuation rings of any ordered field are totally ordered under inclusion, see e.g. [L2, (2.6)], there is a smallest one and a largest one among all convex valuation rings of $k(\alpha)$ dominating $A_{\alpha, \beta}$. In fact, $V_{\alpha, \beta}$ is the smallest; the greatest one will be denoted by $W_{\alpha, \beta}$. Given any one of these valuation rings, say $V$, we write $k_{V}$ for its real closed residue class field, and write $\lambda_{V}$ : $k(\alpha) \rightarrow k_{V} \cup \infty$ for the canonical place. However, in the case of $V_{\alpha, \beta}$ and $W_{\alpha, \beta}$ we write $\mu_{\alpha, \beta}, K$ and $\lambda_{\alpha, \beta}, L$ instead. Given such a valuation ring $V$ we have the diagram

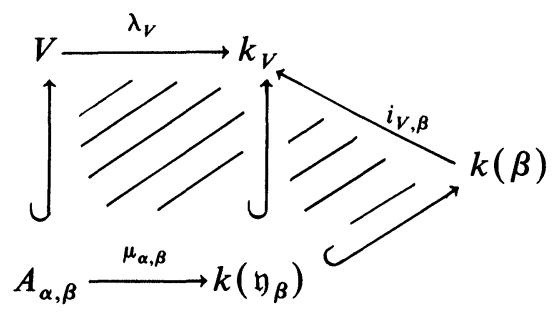

with a uniquely defined monomorphism $i_{V, \beta}$. Note that $k(\beta)$ is a fixed real closure of $k\left(\mathfrak{y}_{\beta}\right)$. If $V=W_{\alpha, \beta}$ we set $i_{V, \beta}=i_{\alpha, \beta}$. We will use the following lemma.

(4.15) Lemma. Given $\alpha \subset \beta$ and $z \in k(\alpha), y \in k(\beta)$. Then the following statements are equivalent:

(i) $x \in W_{\alpha, \beta}$ and $\lambda_{\alpha, \beta}(x)=i_{\alpha, \beta}(y)$.

(ii) For all convex valuation rings $V$ satisfying (4.14), we have $x \in V$ and $\lambda_{V}(x)=i_{V, \beta}(y)$.

Proof. We need only prove (i) $\Rightarrow$ (ii). From valuation theory we get the existence of a place $\mu: L \rightarrow k_{V} \cup \infty$ yielding the following commutative diagram:

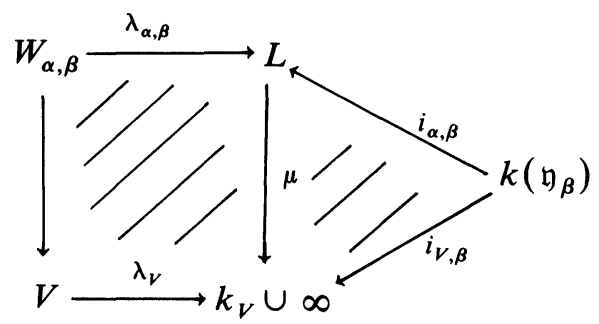

Since $i_{\alpha, \beta}\left(k\left(\mathfrak{y}_{\beta}\right)\right)=\lambda_{\alpha, \beta}\left(A_{\alpha, \beta}\right) \subset \lambda_{\alpha, \beta}(V)$ we see, in view of the fact that $\lambda_{\alpha, \beta}(V)$ is integrally closed, that $i_{\alpha, \beta}(k(\beta))$ is also contained in $\lambda_{\alpha, \beta}(V)$. This leads to the commutative diagram

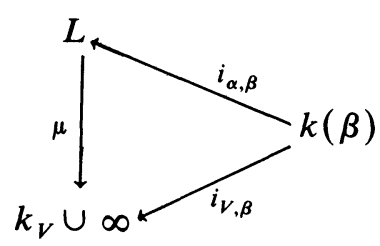


as can be seen by an argument similar to the proof of (2.16). From $i_{\alpha, \beta}(y) \in$ $\lambda_{\alpha, \beta}(V)$ we therefore deduce $i_{V, \beta}(y)=\mu i_{\alpha, \beta}(y)=\mu \lambda_{\alpha, \beta}(x)=\lambda_{V}(x)$. Since $i_{V, \beta}(y) \neq \infty$ we see $x \in V$.

(4.17) Proposition. Let $s$ be a constructible section. Then the following conditions are equivalent:

(i) $s$ is continuous and $s(X)$ is closed in $p^{-1}(X)$.

(ii) For all $\alpha, \beta \in X, \alpha \subset \beta$, we have $T(s(\alpha)) \in W_{\alpha, \beta}$ and $\lambda_{\alpha, \beta}(T(s(\alpha)))=$ $i_{\alpha, \beta}(T(s(\beta)))$.

Proof. (i) $\Rightarrow$ (ii) Suppose $\alpha \subset \beta$. Since $k(\beta)$ is archimedean over $k\left(\mathfrak{y}_{\beta}\right)$ and $y:=T(s(\beta)) \in k(\beta)$ we find $a, b \in A$ with $b(\beta) \neq 0$ and $y^{2}<a(\beta) / b(\beta)$. Consider

$$
U=\left\{\gamma \in \mathscr{R}-\operatorname{Spec} A[T] \mid b(\gamma) \neq 0, T(\gamma)^{2}<\frac{a(\gamma)}{b(\gamma)}\right\} .
$$

$U$ is open, hence, by the continuity of $s$, the set $s^{-1}(U)$ is open. Clearly, $s(\beta) \in U$ and $\beta \in s^{-1}(U)$. Because of $\alpha \subset \beta$ we get $\alpha \in s^{-1}(U)$ from Proposition (2.11). This implies $b(\beta), b(\alpha) \neq 0, T(s(\alpha))^{2}<a(\alpha) / b(\alpha)$ and finally $T(s(\alpha)) \in V_{\alpha, \beta}$. We construct a homomorphism $\varphi: A[T] \rightarrow L$ by requiring $\left.\varphi\right|_{A}=i_{\alpha, \beta} \circ \pi_{\beta}$ and $\varphi(T)=\lambda_{\alpha, \beta}(T(s(\alpha)))$. Setting $\gamma=\varphi_{*}\left(L^{2}\right)$ we get $p \gamma=\beta$ and $s(\alpha) \subset \gamma$. The latter statement follows from the fact that $\lambda_{\alpha, \beta}$ is order-preserving. Now $\gamma \in p^{-1}(X)$ and $\gamma \in \overline{\{s(\alpha)\}}$, hence by assumption, $\gamma \in s(X)$, i.e. $\gamma=s(\beta)$. This means there is also a unique $A$-algebra homomorphism $\psi: A[T] \rightarrow k(\beta)$ with $\left.\psi\right|_{A}=\pi_{\beta}, \gamma=\psi_{*}\left(k(\beta)^{2}\right)$. Now consider $\varphi$ and $i_{\alpha, \beta} \circ \psi=: \varphi^{\prime}$. We shall show $\varphi=\varphi^{\prime}$. This implies

$$
i_{\alpha, \beta}(T(s(\beta)))=\varphi(T)=\lambda_{\alpha, \beta}(T(s(\alpha))),
$$

which is what we have to prove.

Clearly $\varphi_{*}\left(L^{2}\right)=\varphi_{*}^{\prime}\left(L^{2}\right)=\gamma$. The maps $\varphi$ and $\varphi^{\prime}$ induce order-preserving homomorphisms

$$
\left(k\left(\mathfrak{y}_{\gamma}\right), \bar{\gamma}\right) \underset{\bar{\phi}^{\prime}}{\stackrel{\bar{\varphi}}{\rightarrow}} L .
$$

By construction they coincide on the subfield $k\left(\mathfrak{y}_{\beta}\right)$. Since $k\left(\mathfrak{y}_{\gamma}\right)$ is a finite extension-note $T(s(\beta)) \in k(\beta)$ - of $k\left(\mathfrak{y}_{\beta}\right)$, we derive the equality $\bar{\varphi}=\bar{\varphi}^{\prime}$, hence $\varphi=\varphi^{\prime}$. For the converse we first show $s(\alpha) \subset s(\beta)$ provided $\alpha \subset \beta$, $\alpha, \beta \in X$. Pick $F(T) \in s(\alpha)$, then $F(T(s(\alpha))) \geqslant 0$ in $k(\alpha)$ and, since $\lambda_{\alpha, \beta}$ is order-preserving, $i_{\alpha, \beta}(F(T(s(\beta)))) \geqslant 0$ in $L$. Therefore $F(T(s(\beta))) \geqslant 0$ in $k(\beta)$ and $F(T) \in s(\beta)$. To prove the continuity of $s$ we may consider only open constructible subsets $U$ of $p^{-1}(X)$. Then $s^{-1}(U)=p(U)$; showing that $s^{-1}(U)$ is at least constructible as $A[T]$ is obviously finitely presented over $A$, see Theorem (2.15). In order to prove that $s^{-1}(U)$ is also open we apply Proposition (2.11) and check that $s^{-1}(U)$ is closed under generalizations. But this follows immediately from the fact that $\alpha \subset \beta$ implies $s(\alpha) \subset s(\beta)$. It remains to show that $s(X)$ is closed in $p^{-1}(X)$. As observed above, the preimage $s^{-1}(U)$ of any constructible set is again constructible. Therefore $s$ is continuous in the Tychonoff topology. Since $X$, being a constructible set, is 
compact in the latter topology, $s(X)$ turns out to be compact, hence closed in the Tychonoff topology. Thus, it remains to show that $s(X)$ is closed under specialization in $p^{-1}(X)$. So, consider $\alpha \in X, s(\alpha) \subset \gamma, p \gamma=\beta \in X$. We have the valuation ring $V=V_{s(\alpha), \gamma}$ in $k(s(\alpha))=k(\alpha)$ which dominates $A_{s(\alpha), \gamma}$ and $A_{\alpha, \beta}$. Therefore $V_{\alpha, \beta} \subset V$ and

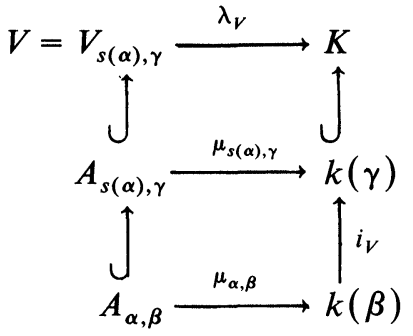

The ordering $\gamma$ is characterized by the two properties $p \gamma=\beta$ and $T(\gamma)=$ $\left.\mu_{s(\alpha), \gamma}(T(s(\alpha)))=\lambda_{V} T(s(\alpha))\right)$. By assumption and (4.15) we also have $\lambda_{V}(T(s(\alpha)))=i_{V}(T(s(\beta)))$. Now this means $\gamma=s(\beta)$, hence $\gamma \in s(X)$. Thus $s(X)$ is closed.

Now the proof of the main Theorem (4.11) is almost complete. It only remains to observe, by using the equivalence of (i) and (ii) in (4.17), that the sum and product of two constructible continuous sections with closed images are again of this type. This is exactly what we have to prove.

We will conclude this section and this paper by several remarks. They illustrate the importance of N. Schwartz' approach, although this can only be hinted at here. The interested reader should not hesitate to consult the appropriate papers [S2, S3, D, DK3].

First of all, let us mention that there exists a sheaf of abstract semialgebraic functions on a constructible set $X \subset \mathscr{R}$-Spec $A$ : Clearly, if $U_{1} \subset U_{2}$ are constructible subsets of $X$ then we have the restriction $\operatorname{res}(s)$ of a section $s$ : $U_{2} \rightarrow \mathscr{R}$-Spec $A[T]$ to $U_{1}$. It is readily checked that $\operatorname{res}(s)$ is in $C\left(U_{1}\right)$ if $s \in C\left(U_{2}\right)$. One further proves that the assignment $U \mapsto C(U)$, where $U$ is open constructible, together with the restriction maps, determines a sheaf of rings on the topological space $X$. Note that the open constructible subsets of $X$ form a basis of the topology of $X$. In fact, this sheaf turns $X$ into a locally ringed space: it is called the structure sheaf of $X$.

Classical algebraic geometry is built up with affine schemes $\operatorname{Spec} A$ as building blocks. Similarly, the constructible subsets together with their structure sheaf may serve as the affine building blocks for a real algebraic geometry in its widest sense. This is done in [S2] where general semialgebraic spaces are introduced. These are locally ringed spaces which are locally isomorphic to constructible sets with their structure sheaves. In this way we obtain the category of abstract semialgebraic spaces in which, as N. Schwartz showed, fibre-products exist. Thus, a quite satisfactory theory may be developed. In particular, H. Delfs and M. Knebusch's theory of "concrete" semialgebraic spaces $X$ over a real closed field $R$ may now be embedded in the somewhat more flexible and comprehensive theory of abstract semialgebraic spaces: Namely, by extending the operation $S \mapsto \tilde{S}, S$ being any semialgebraic set in 
$M(R)$ and $\tilde{S}$ its corresponding constructible set in $\mathscr{R}$-Spec $R[M]$, to any semialgebraic space $X$ in the sense of Delfs-Knebusch, we get an associated abstract semialgebraic space $\tilde{X}$. As demonstrated in $[\mathbf{S 2}, \mathbf{D}]$ several phenomena related to $X$ can be more naturally interpreted in terms of $\tilde{X}$. The most striking example is $N$. Schwartz' solution of the problem of characterizing affine concrete semialgebraic $S$ (i.e. $S$ semialgebraic in some $M(R)$ ) among all concrete semialgebraic spaces. Let us turn to the associated abstract space $\tilde{S}$. If $S$ is affine then $\tilde{S}$ is affine, i.e. $\tilde{S}$ is constructible. In this case $\tilde{S}^{\max }$ (= the set of all closed points) is a Hausdorff space, as seen in the second section. It is this property that is decisive, since $\mathrm{N}$. Schwartz was able to prove the converse: A concrete semialgebraic space is affine iff $\tilde{S}^{\max }$ is a Hausdorff space. A really marvelous theorem!

\section{REFERENCES}

[A] E. Artin, Über die Zerlegung definiter Funktionen in Quadrate, Hamb. Abh. 5 (1927), $100-115$.

[B] E. Becker, Valuations and real places in the theory of formally real fields, in [CT-C-M-R], pp. 1-40.

[Bi] G. Birkhoff, Lattice theory, Amer. Math. Soc., Providence, R.I., 1967.

[Bo1] N. Bourbaki, Topologie générale, Chapter 1, Hermann, Paris, 1951.

[Bo2] _ Algébre commutative, Chapter 2, Hermann, Paris, 1961.

[Br1] L. Bröcker, Positivbereiche in kommutativen Ringen, Abh. Math. Sem. Univ. Hamburg 52 (1982), 170-178.

[Br2] _ Real spectra and distributions of signature, in [CT-C-M-R], pp. 249-272.

[Br3] _ Minimale Erzeugung von Positivbereichen, Geom. Dedicata 16 (1984), $335-350$.

[Br4] , Spaces of orderings and semialgebraic sets, Proc. Conf. on Quadratic and Hermitian Forms (Hamilton 1983), CMS Conf. Proc., vol. 4, Amer. Math. Soc., Providence, R. I., 1984.

[Bru1] G. W. Brumfiel, Partially ordered rings and semialgebraic geometry, Cambridge, 1979.

[Bru2] __ Witt rings and K-theory, Rocky Mountain J. Math. 14 (1984), 733-765.

[BS] L. Bröcker and J.-H. Schinke, On the L-adic spectrum, preprint, 1986.

[C] M. Coste, Ensembles semi-algébriques, in [CT-C-M-R], pp. 109-138.

[CC] M. Coste and M.-F. Coste-Roy, Topologies for real algebraic geometry, in Topos Theoretic Methods in Geometry (A. Kock, ed.) Various Publ. Series 30, Aarhus Universitet, 1979.

[CC1] __ La topologie du spectre réel, Contemporary Math. 8 (1982), 27-60.

[CR] M.-F. Coste-Roy, Spectre réel d' un anneau et topos étale réel, Thèse Univ. Paris-Nord, 1980.

[CT] J.-L. Colliot-Thélène, Variantes du Nullstellensatzz réel et anneaux formellement réels, in [CT-C-M-R], pp. 98-108.

[CT-C-M-R] J.-L. Colliot-Thélène, M. Coste, L. Mahé and M.-F. Roy, (eds.), Proc. Conf. on "Géométrique Algébrique Réele et Formes Quadratiques", Rennes 1981, Lecture Notes in Math., vol. 959, Springer-Verlag, Berlin and New York, 1982.

[D] H. Delfs, The homotopy axiom in semialgebraic cohomology, J. Reine Angew. Math. 355 (1985), 108-128.

[De] C. Delzell, A finiteness theorem for open semi-algebraic sets with applications to Hilbert's 17th problem, Contemporary Math. 8 (1982), 79-97.

[Dr] L. v. d. Dries, Some applications of a model theoretic fact to (semi-)algebraic geometry, Indag. Math. 44 (1982), 397-401.

[Du] D. W. Dubois, Real commutative algebra, I. Places, Rev. Mat. Hisp.-Amer. 39 (1979), 57-65.

[DE] D. Dubois and G. Efroymson, Algebraic theory of real varieties, in Studies and Essays, presented to Yu-Why Chen on his 60th birthday, Taipei 1970, pp. 107-135. 
[DK1] H. Delfs and M. Knebusch, Semialgebraic topology over a real closed field. II, Math. Z. 178 (1981), 175-213.

[DK2] , On the homology of algebraic varieties over real closed fields, J. Reine Angew. Math. 335 (1982), 122-163.

[DK3] H. Delfs and M. Knebusch, Locally semialgebraic spaces, Lecture Notes in Math., vol. 1173, Springer-Verlag, Berlin and New York, 1985.

[DR] D. W. Dubois and T. Recio (eds.), Ordered fields and real algebraic geometry, Contemporary Math. 8 (1982).

[K] I. Kaplansky, Commutative rings, Chicago, 1970.

[Kn] M. Knebusch, An invitation to real spectra, Proc. Conf. on Quadratic and Hermitian Forms (Hamilton, 1983), CMS Conf. Proc., vol. 4, Amer. Math. Soc., Providence, R. I., 1984.

[L1] T. Y. Lam, Orderings, valuations and quadratic forms, CBMS Regional Conf. Ser. in Math. no. 52, Amer. Math. Soc., Providence, R.I., 1983.

[L2] _ An introduction to real algebra, Rocky Mountain J. Math. 14 (1984), 767-814.

[La] S. Lang, Algebra, 2nd ed., Addison-Wesley, Reading, Mass., 1967.

[Lo] S. Lojasiewicz, Ensembles semi-analytiques, multigraphié I.H.E.S., 1965.

[Mc] K. McKenna, New facts about Hilbert's 17th problem, Lecture Notes in Math., vol. 498, Springer-Verlag, Berlin and New York, 1975, pp. 220-230.

[P] A. Prestel, Lectures on formally real fields, Lecture Notes in Math., vol. 1093, Springer-Verlag, Berlin and New York, 1984.

[PC] S. Priess-Crampe, Angeordnete Strukturen: Gruppen, Körper, projektive Ebenen, Springer, Berlin, Heidelberg and New York, 1983.

[PR] A. Prestel and P. Roquette, Formally p-adic fields, Lecture Notes in Math., vol. 1050, Springer-Verlag, Berlin and New York, 1984.

[R] M.-F. Roy, Faisceau structural sur le spectre réel et fonctions de Nash, in [CT-C-M-R], pp. 406-432.

[S] G. E. Sacks, Saturated model theory, W. A. Benjamin, Inc., Reading, Mass., 1972.

[S1] N. Schwartz, Der Raum der Zusammenhangskomponenten einer reellen Varietät, Geom. Dedicata 13 (1983), 361-397.

[S2] _ Real closed spaces, Habilitationsschrift, München, 1984.

[S3] _ Real closed rings, preprint, 1985.

[St] G. Stengle, A Nullstellensatz and a Positivstellensatz in semialgebraic geometry, Math. Ann. 207 (1974), 87-97.

[W] B. L. v. d. Waerden, Algebra. I. 5th ed., Springer-Verlag, Berlin, Göttingen, Heidelberg, 1960.

[Wh] H. Whitney, Elementary structure of real algebraic varieties, Ann. of Math. 66 (1957), $545-556$.

[ZS] O. Zariski and P. Samuel, Commutative algebra. II, New York, 1960.

FB Mathematik, Universität Dortmund, Postfach 5005 00, 4600 Dortmund 50, Federal REPUBLIC OF GERMANY 